\title{
PROPOSTA DE UM GRÁFICO DE CONTROLE X-BAR MODIFICADO COM LIMITES ASSIMÉTRICOS E COM AMOSTRAGEM MÚLTIPLA
}

\section{PROPOSED MODIFIED X-BAR CONTROL CHART WITH ASYMMETRIC LIMITS AND MULTIPLE SAMPLING}

\author{
Naijela Janaina da Costa* E-mail: naijelajanaina@gmail.com \\ Carlos Ivan Mozambani* E-mail: cmozambani@gmail.com \\ Celso Luiz Gonçalves* E-mail: celso luiz goncalves@yahoo.com.br \\ Pedro Carlos Oprime* E-mail: pedro.oprime@gmail.com \\ *Universidade Federal de São Carlos (UFSCar), São Carlos, SP
}

\begin{abstract}
Resumo: Neste artigo encontra-se uma proposta de gráfico de controle modificado com limites assimétricos que utiliza amostragem múltipla para decidir o estado de controle dos processos. Dois temas correlatos estabeleceram as bases teóricas da proposta apresentada: projetos de gráficos de controle com parâmetros variados $(\mathrm{pV})$; e, gráficos de controle com parâmetros fixos $(\mathrm{pF})$. O uso de amostragem múltipla melhora o poder de detecção de causas especiais e minimiza o risco de parar o processo indevidamente por ocorrência do erro tipo I (falso positivo). Propõe-se então, a utilização de um gráfico de controle estatístico com limites assimétricos utilizando-se um procedimento de amostragem não linear (não fixo) com três possíveis regiões de decisão. A proposta considera a possibilidade de reamostragem do processo. Os efeitos das estimativas de parâmetros estatísticos também foram considerados na análise do desempenho do gráfico proposto. A métrica de desempenho considerada foi a esperança matemática sobre o número médio de amostras até detectar um ponto fora dos limites de controle. Métodos numéricos foram utilizados para encontrar os limites estatísticos de controle para um erro tipo I de 0,27\%.
\end{abstract}

Palavras-chave: Amostragem múltipla. Carta de controle. Limites assimétricos. Parâmetros variados.

Abstract: In this paper we present a proposal of statistical control chart modified with asymmetric limits that use multiple sampling to decide the state of control of the process. Two related themes established the theoretical bases of the presented proposal: control charts designs with variable parameters $(\mathrm{pV})$; and, control charts with fixed parameters $(\mathrm{pF})$. The use of multiple sampling improves the power of detection of special causes and minimizes the risk of stopping the process unduly by the occurrence of type I (false positive) error. It is proposed to use a statistical control chart with asymmetric limits using a non-linear (non-fixed) sampling procedure with three possible decision regions. The proposal considers the possibility of resampling the process. The effects of statistical parameter estimates were also considered in the performance analysis of the proposed chart. The performance metric considered was the mathematical expectation on the average number of samples until detecting a point outside the control limits. Numerical methods were used to find the statistical control limits for a type I error of $0.27 \%$.

Keywords: Multiple sampling. Control chart. Asymmetric limits. Variable parameters

\section{INTRODUÇÃO}

A carta ou gráfico de controle estatístico é uma das ferramentas amplamente utilizada pelas empresas no Controle Estatístico de Processo (CEP). Em 1920 elas 
foram introduzidas por W. Schewhart, no qual, as cartas do tipo $\bar{X}$ e $R / S$ são as mais conhecidas. O fundamento teórico dos gráficos de controle está em lidar com as variáveis de qualidade de um produto ou processo, sendo usualmente necessário mantê-las estáveis ao longo do tempo. Para tal condição de estabilidade é necessário monitorar a posição central (ou de locação) e a dispersão (ou escala), por meio de estimadores adequados, como a média $\mu$ e o desvio padrão $\sigma$, respectivamente (MONTGOMERY, 2004).

A análise sobre o estado de controle das medidas de posição central e de escala são realizadas por meio de diretrizes e procedimentos amostrais previamente planejados e representativos do processo. Como a decisão do estado de controle baseia-se em análises estatísticas amostrais, há consequentemente riscos de erros de decisão, ou seja: afirmar que o processo não está sob controle quando na realidade está (erro de decisão tipo I), ou; afirmar que o processo está sob controle quando na realidade não está (erro tipo II).

A decisão sobre qual tipo (design) de gráficos de controle a ser utilizado geralmente baseia-se no maior Número Médio de Amostras (NMA) tomadas do processo até a detecção no gráfico de um ponto fora de controle, quando o processo estiver sob controle, e, por outro, deseja-se um menor número de amostras tomadas para quando o processo estiver fora de controle. Em termos estatísticos, a decisão sobre o estado de controle de um processo por meio de gráficos estatísticos baseiase nos níveis de riscos de decisão tipo I e II, normalmente denominados de erros $\alpha \mathrm{e}$ $\beta$, respectivamente. Esses níveis de riscos estão correlacionados diretamente com as estimativas dos parâmetros estatísticos utilizados nos cálculos dos limites de controle feitos na fase de implantação (fase I), denominada de fase retrospectiva, e na fase de monitoramento (fase II), denominada de prospectiva (MONTGOMERY; RUNGER, 2009).

Utilizam-se basicamente três parâmetros para a construção desses gráficos: o tamanho da amostra $n$, o número de amostras $m$ (utilizado na fase de estimativa dos parâmetros estatísticos da fase I) e a constante $k$ (geralmente $k=3$ ), que em condições ideais representa um erro $\alpha=0,27 \%$. Usualmente esses parâmetros são mantidos constantes, e quando isso ocorre são mencionados na literatura como cartas ou gráficos de controle com parâmetros fixos (pF) (MONTGOMERY, 2004). 
As características de gráficos de controle apresentadas até aqui referem-se ao modelo clássico proposto por Shewhart. No entanto, surge em alguns trabalhos mudanças que melhoram o desempenho dessas cartas de controle. Autores como Costa (1998a); Costa (1998b); Costa e De Magalhães (2005); De Magalhães, Epprecht e Costa (2001); De Magalhães e Moura Neto (2005); e, PeñabaenaNiebles et al. (2014) desenvolveram cartas com parâmetros variados (pV), que, segundo eles, apresentaram desempenho estatístico e resultado econômico superiores as cartas de controle com pF. As escolhas dos parâmetros dos designs das cartas de controle são baseadas nos erros tipo I ( $\alpha$ ) e tipo II ( $\beta$ ), nos custos que envolvem todo o procedimento de controle e nos efeitos decorrentes de decisões equivocadas.

O erro tipo I é um parâmetro usualmente fixado, no entanto se torna uma fragilidade da abordagem clássica, pois se pressupõe que os parâmetros estatísticos sejam conhecidos, o que na prática nem sempre ocorre. Ao fixar $k=3$ é suposto que $\alpha$ seja igual a $0,27 \%$, porém quando os parâmetros estatísticos, como a média $\mu$ e $\sigma$ são estimados, o erro tipo I é maior que 0,27\%. Essa questão é denominada como efeito da estimativa de parâmetros e foi considerada em diversos outros estudos (CASTAGLIOLA; CELANO; CHEN, 2009; CASTAGLIOLA; CELANO; FICHERA, 2013; CASTAGLIOLA; MARAVELAKIS, 2011; JENSEN et al., 2006; OPRIME; GANGA, 2015).

Embora os estudos citados apresentem contribuições significativas para modelos econômicos de gráficos de controle pela abordagem de variação do número de amostras e tamanho da amostra, bem como dos efeitos da estimativa de parâmetros no desempenho dos gráficos de controle, os mesmos estudos não consideraram o modo como a média é afetada por causas identificáveis e como isso implica em magnitudes distintas sobre os efeitos na qualidade do produto. Em outras palavras, estes estudos não consideram, por exemplo, os casos onde o desvio positivo na média pode ter menor efeito na qualidade do produto que desvios negativos.

Com o objetivo de melhorar o desempenho dos gráficos de controle X-bar tradicional, em termos da sua capacidade em se detectar efeitos significativos na média, bem como o de reduzir os custos de amostragem, é proposto neste artigo um novo design de gráficos de controle com limites assimétricos com três regiões de 
decisão sobre o processo: região $\mathrm{M0}$ - que indica o estado de controle, ou seja, aceita-se o processo como adequado; região I - sem decisão ou de dúvida, o que implica em nova amostra; e região M1 - que indica o estado de fora de controle do processo, ou seja, para-se o processo e busca-se as causas especiais. Portanto, implica-se no aumento no tamanho da amostra; ou redução do intervalo entre amostragem; ou ainda, afrouxamento no tamanho da amostra. Essa abordagem permite a construção de gráficos de controle mais econômicos e com maior robustez similar aos trabalhos de, De Magalhães, Epprecht e Costa (2001), e Schoonhoven e Does (2012). A diferença que, para resolver o problema proposto utilizam-se dois valores diferentes da constante $K, k_{1}, k_{2}$ para o cálculo dos limites de controle inferior e superior (LIC e LSC), respectivamente.

Outra diferença apontada neste artigo é a possibilidade de reamostragem do processo (amostragem múltipla). Costa (1998b) ressalta que esta abordagem minimiza as paradas e o erro tipo I, dependendo da região onde a estatística amostral caia e considerando que os parâmetros estatísticos sejam desconhecidos.

Desta forma o presente trabalho está organizado em quatro seções além desta introdução. A segunda seção apresenta um quadro abordando os diferentes temas de cartas de controle, bem como o processo de construção das cartas tipo $\bar{X}$ e $R$ com parâmetros fixo (pF) e com parâmetros variados (pV). Na terceira encontrase o método de pesquisa e na quarta seção a análise de desempenho por meio do Número Médio de Amostras (NMA) que conta com um framework do método proposto buscando melhor compreensão em relação ao processo de tripla amostragem. Por fim as principais considerações encontram-se na seção 5 deste trabalho.

\section{REVISÃO DE LITERATURA}

Nesta seção é apresentada uma revisão de literatura contendo temas relacionados ao Controle Estatístico de Processos, a fim de explorar conceitos e aplicações. Foram analisados artigos de periódicos com revisão por pares, congressos, teses, dissertações e livros. No processo de busca e análise dos artigos foram utilizados os portais Web of Science e Scopus. Estas bases de dados são portais mantidos por grandes editores científicos que indexam uma série de 
publicações científicas e tecnológicas em todas as áreas do conhecimento, oferecendo um número significativo de obras publicadas. Foram utilizados os seguintes parâmetros de pesquisa: $X$ control charts AND asymmetric limits; $X$-bar charts AND multiple sampling; $X$ control charts AND economic design.

Alguns trabalhos foram selecionados e encontram-se no Quadro 1. Observase que os artigos listados abordam diferentes temas de cartas de controle de Shewhart. Neste trabalho, foram incluídos limites de controle assimétricos observando que há situações práticas onde o sinal de mudança na média tem diferentes impactos na qualidade dos produtos e processos. As hipóteses são de que o uso de múltiplas amostras minimiza a ocorrência dos erros tipo I e II.

Quadro 1 - Alguns trabalhos encontrados na literatura (continua)

\begin{tabular}{|c|l|}
\hline Autor(es)/Ano & \multicolumn{1}{|c|}{ Foco de análise } \\
\hline Avinadav, & O objetivo deste estudo foi minimizar o custo de qualidade total esperado por lote \\
Perlman e Cheng & em um processo de fabricação. Para encontrar o tamanho ideal da amostra e os \\
$(2016)^{1} /$ Hong & limites de controle, um modelo de cadeia de Markov foi formulado. O impacto dos \\
Kong $^{2} /$ & parâmetros do modelo e suas interações na solução ótima foram explorados \\
Engenharia $^{3}$ I & através da análise de variância em 6.561 exemplos numéricos em um projeto \\
Scopus e Web of & fatorial. Finalmente, um exemplo de aplicação real demonstra a eficácia do \\
Science ${ }^{4}$ & modelo proposto e da heurística.
\end{tabular}


Quadro 1 - Alguns trabalhos encontrados na literatura

(conclusão)

Chen e Kuo $(2010)^{1} /$ Taiwan $^{2}$

/ Engenharia e

Ciências da

Decisão ${ }^{3} /$

Scopus e Web of Science ${ }^{4}$

Este artigo examinou o desempenho relativo de limites simétricos e assimétricos. O artigo comparou o Número Médio de Amostras (NMA) fora de controle para limites simétricos e assimétricos. Para os gráficos $\bar{X}$ e $R$, o impacto da escolha do limite de controle (simétrico ou assimétrico) depende de dois fatores: a inclinação da estatística de gráficos (média da amostra $X$ para o gráfico $\bar{X}$ e intervalo de amostra $R$ para o gráfico $R$ ); e, a direção. Quando a estatística de gráficos tem uma distribuição esquerda-inclinada, o resultado é invertido. Embora nenhum dos limites de controle domine para uma mesma população distorcida, os limites assimétricos são mais robustos à mudança na média ou variação do processo.

Este estudo propôs uma abordagem que considera simultaneamente as propriedades de custo e qualidade pelo valor mínimo do custo esperado por hora, que é restringido pelo valor máximo do erro tipo I e pelo valor mínimo de potência para determinar três parâmetros (tamanho; intervalo de amostragem entre amostras sucessivas; e, os limites de controle) quando um gráfico $\bar{X}$ supervisiona um processo de fabricação com uma taxa de risco aumentada e as medidas dentro da amostra sendo correlacionadas. Como resultado, o pressuposto do mecanismo de falha de Poisson para processos em que o desgaste da máquina ocorre ao longo do tempo não é apropriado.

$(2010)^{1} /$ Taiwan $^{2}$

/ Ciências da

Computação ${ }^{3} /$

Scopus $^{4}$

Chen $(2009)^{1} /$

Taiwan $^{2} /$

Engenharia $^{3}$ /

Scopus $^{4}$

Neste trabalho foi aplicado o gráfico de controle $\overline{\bar{X}}$ no modelo EMQ (Economic Manufacturing Quantity) modificado. O custo total mínimo da sociedade, incluindo o custo de controle de estoque e custo de qualidade, necessita ser obtido para ter a quantidade econômica de fabricação e os parâmetros ótimos de limites de controle da carta de controle $\bar{X}$. A qualidade do produto foi mensurada pela adoção da função de perda de qualidade quadrática assimétrica de Taguchi.

Chen e Kuo $(2007)^{1} /$ Taiwan $^{2}$ / Engenharia; e Negócios, Gestão e Contabilidade ${ }^{3}$ / Scopus e Web of Science $^{4}$ Esta pesquisa teve por intuito comparar o desempenho do gráfico $\overline{\bar{Z}}$ para os limites simétricos e assimétricos. Comparou-se o NMA fora de controle para limites simétricos e assimétricos enquanto mantiveram-se os mesmos valores de NMA em controle. O estudo constatou que se a característica de qualidade tem uma distribuição direita distorcida, os limites simétricos obtêm melhores resultados do que os limites assimétricos quando a média do processo se desloca para um valor maior. Ressalta-se que, mesmo quando a característica de qualidade tem uma distribuição distorcida, a escolha de limites simétricos ou assimétricos depende das direções da mudança e da inclinação.

De Magalhães, Epprecht e Costa $(2001)^{1} /$ Brasil $^{2} /$

Engenharia;

Economia;

Econometria e

Finanças ${ }^{3}$ I

Scopus eWeb of Science $^{4}$

Costa $(1998 b)^{1} /$ Brasil $^{2}$ /

Engenharia e

Ciências da

Decisão ${ }^{3}$ /

Scopus eWeb of Science 4

Neste trabalho, foi desenvolvido um modelo econômico para os gráficos de controle $\bar{X}$ contendo todos os parâmetros variados de forma adaptativa, ou seja, em tempo real, considerando a informação da amostra atual. No modelo proposto, cada um dos parâmetros de projeto pode assumir dois valores como uma função das informações de processo mais recentes. A função de custo é derivada e fornece um dispositivo para seleção ótima de parâmetros de projeto. Através de um exemplo numérico, é possível prever as economias que o modelo desenvolvido possivelmente fornece.

Este artigo estende os estudos aos processos que são monitorados pelos gráficos $\bar{X}$ e $R$. Quando os valores de $\bar{X}$ e $R$ caem na região central o controle é relaxado porque se deve esperar um pouco mais para tomar a próxima amostra e/ou tomar uma amostra menor. Quando os valores de $\bar{X}$ ou $R$ caem na região de aviso o controle é apertado porque se deve esperar menos para tomar a próxima amostra e a mesma será maior do que o habitual. Os limites de ação também são tornados variáveis. Assim, este artigo esboça os limites da ação (para ambos os gráficos) que são mais largos do que o normal.

Fonte: Elaborado pelos autores (2017)

${ }^{1}$ Autor(es)/ano - ${ }^{2}$ País/território - ${ }^{3}$ Área de estudo $-{ }^{4}$ Base de dados 


\subsection{Controle Estatístico do Processo (CEP)}

De Vries e Reneu, (2010), Ferreira et al. (2011), Korzenowski (2012), Marshall Junior et al. (2003) e Yang et al. (2002) definem o CEP como um conjunto de ferramentas de monitoramento, controle e melhoria na qualidade dos processos por meio de análises estatísticas. A principal ferramenta utilizada pelo CEP é o gráfico/carta de controle, que usualmente monitora uma variável de qualidade tanto pelo seu valor médio quanto pela sua variabilidade utilizando parâmetros com valores fixos $(\mathrm{pF})$.

\subsection{Cartas tipo $\bar{x}$ e $R$ com parâmetros fixos (pF)}

Em cartas de controle do tipo $\bar{X}$ e $R$ pF são denotados pelos parâmetros $n_{0}, m_{0}, k_{0}, L_{0}$ e $\delta_{0}$. Considere: $n_{0}$ (tamanho de amostras); $m_{0}$ (número de amostras); $k_{0}$ (constante); $L_{0}$ (limites); e, $\delta_{0}$ (número de desvios em relação a média populacional quando o processo está fora de controle, podendo ser positivo ou negativo).

Considera-se que os LSC e LIC são dados por $\mu_{0}-k_{0} \sigma / \sqrt{n_{0}}$ e a linha central $\mu_{0}$ conforme mostra a Figura 1. A relação $k_{0} \sigma / \sqrt{n_{0}}$ é uma constante que depende de $n_{0}$ e que pode ser encontrada tabulada em tabelas (MONTGOMERY, 2004).

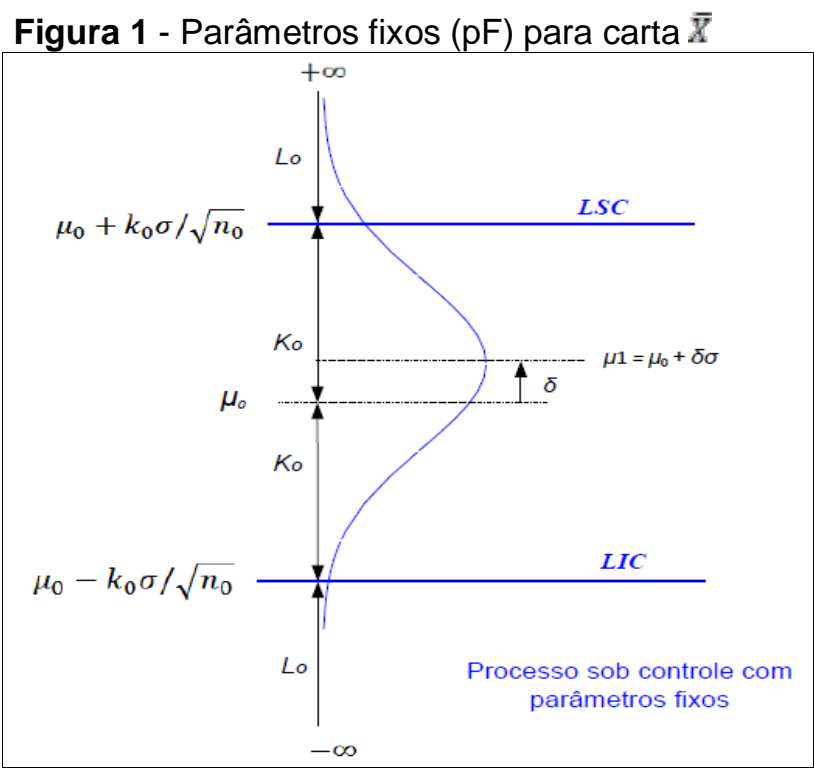

Fonte: Adaptado de Montgomery (2004) 


\subsubsection{Número Médio de Amostras para o gráfico $\bar{x}$}

Normalmente os parâmetros estatísticos são estimados e o uso dessas estimativas presumidamente gera erros no cálculo da estimativa dos limites de controle na fase I (os limites de controle são variáveis aleatórias) que afetam o desempenho dos gráficos de controle na fase II. O desempenho de um gráfico de controle é avaliado de acordo com a sua capacidade de detectar causas especiais e com o menor erro tipo I. Ressalta-se que a decisão sobre o estado de controle de um processo por meio de gráficos estatísticos baseia-se nos níveis de riscos de decisão tipo I e II, normalmente denominados de erros $\alpha$ e $\beta$, respectivamente.

Nos processos industriais, os erros $\alpha$ e $\beta$ estão presentes em várias atividades e decisões da função qualidade, por exemplo, no lançamento de novos produtos, na aprovação da produção, na aprovação de lotes recebidos de fornecedores, na aprovação e validação de sistemas de medição, e na liberação de lotes para clientes (JURAN; GRYNA, 1981). Essa abordagem é tradicionalmente utilizada na elaboração dos planos de amostragem de aceitação lote a lote ou inspeção contínua (MONTGOMERY; RUNGER, 2009). O mesmo teste estatístico pode ser realizado nos testes de capabilidade de processos na compra de um novo equipamento de produção, nos testes de confiabilidade para validar produtos e processos, nos testes de produto final e nas decisões sobre a qualidade do processo por meio de gráficos de controle (AIAG, 1991; MONTGOMERY; RUNGER, 2009).

As decisões aqui descritas e normalmente realizadas na indústria manufatureira baseiam-se nas técnicas de testes de hipóteses estatísticos, onde $\mathrm{H}_{0}$ é hipótese nula, que em termos práticos está relacionada à teoria econômica da decisão, ou seja, significa que ao rejeitar essa hipótese os impactos econômicos ou práticos serão significativos para o tomador de decisão. De fato, a hipótese nula é um ponto de partida para a aceitação da hipótese alternativa, $\mathrm{H}_{1}$, que por certo é a mais importante em determinadas situações. Um exemplo para esta situação seria um produtor de componentes para a indústria automobilística que não deseja reprovar um lote de peças considerado aceitável. Deste modo, na teoria dos testes de hipóteses estatísticos define-se o erro tipo I como a probabilidade de se rejeitar a hipótese $\mathrm{H}_{0}$ quando de fato ela é verdadeira. Assim, as probabilidades $\alpha$ e $\beta$ dos erros tipo I e II são denominados, respectivamente, risco do produtor e risco do 
consumidor. O $\alpha$ é o risco do produtor, de rejeitar um lote com nível aceitável de qualidade, e $\beta$ é o risco do consumidor de aceitar um lote com nível inaceitável de qualidade.

Por conseguinte, o desempenho na fase II é avaliado pelo Número Médio de Amostras (NMA), que é influenciado pelos erros inerentes de estimativa dos parâmetros estatísticos monitorados nos gráficos de controle (CASTAGLIOLA; CELANO; CHEN, 2009; CASTAGLIOLA; MARAVELAKIS, 2011).

O NMA pode ser interpretado como uma medida de equilíbrio do erro tipo I, que representa o controle excessivo ou alarme falso, quando o processo está sob controle, e pelo erro tipo II, que é o controle inadequado, ou incapacidade de detectar o estado fora de controle do processo. O NMA estima o número médio de amostras extraídas do processo até obter um sinal positivo e essa medida estatística é intuitiva, o que gera certa dificuldade em interpretá-la. Se as observações plotadas no gráfico de controle são independentes, então o número de pontos que devem ser plotados até o primeiro ponto exceder os limites de controle é uma variável aleatória geométrica com parâmetro $p$. A média dessa distribuição é $1 / p$, que é 0 comprimento médio das corridas (MONTGOMERY, 2004). O NMA pode ser expresso como:

$$
N M A=\frac{1}{p(\text { um ponto cair alem dos limites de controle })}
$$

ou

$$
N M A_{0}=\frac{1}{p(\text { Erro Tipo I })}=\frac{1}{\alpha} \quad \text { para processos sob controle }{ }^{n}
$$

$\mathrm{e}$

$$
N M A_{1}=\frac{1}{p(1-\beta)} \quad \quad \text { "para processos fora de controle" }
$$

onde $p$ expressa a probabilidade de ocorrência do evento.

\subsubsection{Cartas de Controle tipo $\overline{\mathrm{X}}$ e R com parâmetros variados (pV)}

Há vários autores que atualmente abordam sobre CEP por meio de cartas de controle com parâmetros variados contrapondo as cartas de controle descritas 
acima. Pode-se considerar o trabalho de Reynolds et al. (1988) como pioneiro do pensamento de variação de parâmetros para cartas de controle. O autor introduziu a ideia de variação de intervalo amostral (Varying the Sampling Interval- VSI) para cartas tipo $\bar{X}$. Futuramente o VSI foi expandido para outras cartas como CUSUM e EWMA (REYNOLDS; AMIN; ARNOLD, 1990; SACCUCCI; AMIN; LUCAS, 1992).

Sequencialmente, a ideia de variação do tamanho da amostra (Varying the Sample Size - VSS) foi estudada por Park e Choi (1992), oferecendo significativa contribuição aos estudos de variação paramétrica. Prabhu, Montgomery e Runger (1994) contribuíram com o estudo de variação dos dois parâmetros, intervalo amostral e tamanho da amostra (Variable Sample Size and Sampling Interval VSSI). Para uma interessante revisão e evolução consulte Costa (1998a) e De Magalhães, Epprecht e Costa (2001).

Costa (1998b) elaborou uma proposta de carta de controle com 3 regiões: sob controle, aviso ("warning") e ação; para gráficos $\bar{X}$ e $R$, variando 4 parâmetros: $n$ o tamanho da amostra; $m$ o intervalo amostral; $k$ largura da faixa do limite de ação para o gráfico $\bar{X}$; e, $k_{R}(n)$ determinando a largura da faixa do limite de ação para o gráfico $R$. Desta forma o autor explorou a variação dos intervalos de amostras e comparou com gráficos $\bar{X}$ e $R$ com pF. Conforme seu estudo, se um determinado ponto cai na região central (sob controle) o intervalo amostral é expandido ("relaxed"), ou seja, há diminuição de coletas de amostra. Se um dado ponto cai na região de aviso, o controle é estreitado, diminuindo o intervalo entre amostras. Por fim, se determinado ponto cair na região de ação, o processo é parado para detecção das possíveis causas. O autor apresenta um esquema com pV que melhora substancialmente o desempenho do conjunto de cartas tipo $\bar{X}$ e $R$. Por exemplo, para um tamanho de amostra comum (3 a 6 unidades) e uma detecção de mudança de $0,5 \delta$, a proposta de $\mathrm{pV}$ é três vezes mais rápida na detecção do que para esquemas tradicionais com pF e duas vezes mais rápida que outros esquemas tais como VSSI.

Desta forma, elencando os estudos mencionados, assume-se que em determinado processo é empregado as cartas tipo $\bar{X}$ e $R$ com parâmetros variados para observação de características da qualidade de interesse $X$, normalmente distribuídas com média e desvio padrão, $\mu$ e $\sigma^{2}$, respectivamente. Pressupondo que o processo se inicie sob controle $\left(\mu=\mu_{0}\right.$ e $\sigma=\sigma_{0}$ ) e que após algum tempo o 
processo sofre uma mudança de $\mu_{0}$ para $\mu_{1}=\mu_{0}+\delta \sigma$, com $\delta>0$, e/ou desvio padrão mude de $\sigma_{0}$ para $\sigma_{1}=\gamma \sigma_{0}, \operatorname{com} \gamma>1$.

Para o uso da carta de controle devem-se especificar os parâmetros, tais como $n, m, k$, os limites para a região de indiferença $(L)$, e, o número de desvios em relação à média populacional $(\delta)$. $O$ design de parâmetro de cartas tipo $\bar{X}$ e $R$ com $\mathrm{pV}$ em casos gerais podem ser diferentes para cada valor amostrado (DE MAGALHÃES; EPPRECHT; COSTA, 2001). No modelo proposto neste trabalho, os parâmetros podem assumir dois valores relativos aos coeficientes de limites de controle $\left(k_{1}\right.$ e $\left.k_{2}\right)$ e limites para a região de indiferença $\left(L_{1}\right.$ e $\left.L_{2}\right)$. A Figura 2 evidencia os referidos parâmetros.

Logo, os limites estabelecidos para cada região são: a região M0 estará contida entre o LSC $_{1}$ (Limite superior de Controle) e o LIC 1 (Limite Inferior de Controle); a região I, denominada pelo parâmetro $L_{1}$, estará contida acima do $\mathrm{LSC}_{1} \mathrm{e}$ abaixo do $\mathrm{LSC}_{2}$; já para o parâmetro $L_{2}$, a região estará entre o $\mathrm{LIC}_{1}$ e $\mathrm{LIC}_{2}$. Por último a região M1 é definida pelos pontos que caírem acima do $\mathrm{LSC}_{2}$ e abaixo do $\mathrm{LIC}_{2}$, como demonstra a Figura 2.

Figura 2 - Limites de controle propostos

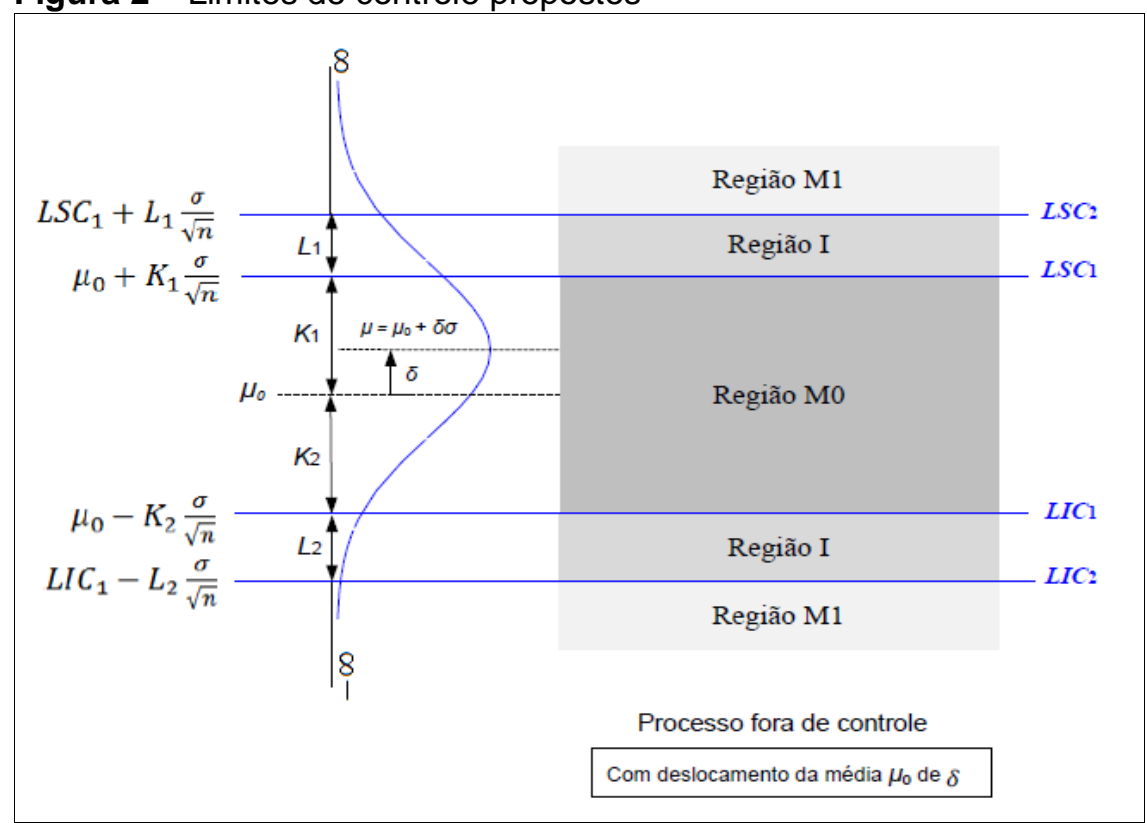

Fonte: Elaborado pelos autores (2017)

Os limites de controle para a média amostral para amostras fixas de tamanho $n$ de cada região quando a média é conhecida são obtidos por: 
- Região MO: $\bar{X} \in\left[L I C_{1} ; L S C_{1}\right]$, onde $L I C_{1}=\mu_{0}-K_{2} \frac{\sigma}{\sqrt{n}}, L S C_{1}=\mu_{0}+K_{1} \frac{\sigma}{\sqrt{n}}$;

- Região I: $\bar{X} \in\left[L I C_{2}-L I C_{1} ; L S C_{2}-L S C_{1}\right]$, onde $L I C_{2}=L I C_{1}-L_{2} \frac{\sigma}{\sqrt{n}} \mathrm{e}$ $L S C_{2}=L S C_{1}+L_{1} \frac{\sigma}{\sqrt{n}}$

- Região M1: $\bar{X} \in\left[-\infty, L I C_{2} ; L S C_{2}, \infty\right]$.

Para o caso quando a média é desconhecida e o desvio padrão é conhecido, a média é estimada na fase I, onde $m$ amostras de tamanho $n$ são extraídas. Para esses casos tem-se que obter as probabilidades da média amostral na fase II cair em cada uma das regiões. Dado que $\left(X_{i, 1}, \ldots, X_{i, n}\right), i=1,2, \ldots, m$ são variáveis aleatórias $X_{i, j} \sim \operatorname{Normal}\left(\mu_{0} ; \sigma_{0}\right)$. A média $\mu_{0}$ é estimada por:

$$
\overline{\bar{x}}=\frac{1}{m n} \sum_{j=1}^{m} \sum_{i=1}^{n} x_{i j}
$$

Na fase II, amostras $\left\{X_{i, 1}, X_{i, 2}, \ldots, X_{i, n}\right\}$ de tamanho $n$ são retiradas do processo. $O$ erro amostral $\overline{\bar{X}}-\mu_{0}=\mathrm{W} \frac{\sigma_{0}}{\sqrt{m, n}}$ é considerado no desenvolvimento do cálculo das probabilidades. A derivação do modelo de probabilidade da média amostral calculada a partir de amostras tomadas na fase II e estarem fora dos limites de controle $\left(L I C_{2}\right.$ e $\left.L S C_{2}\right)$ é obtida por:

$\bar{X} \in\left[-\infty, L I C_{2} ; L S C_{2}, \infty\right]=1-\left[P\left(\bar{x} \in\left[L I C_{1} ; L S C_{1}\right]\right)+P\left(\bar{X} \in\left[L I C_{2}-L I C_{1} ; L S C_{2}-\right.\right.\right.$ $\left.\left.\left.L S C_{1}\right]\right)\right]$

Entretanto, o NMA, ou seja, o número esperado de amostras tomadas antes que o deslocamento seja detectado, possui a característica de uma distribuição geométrica e com probabilidade $p(\delta, m, n)$, e pode ser descrita como:

$$
N M A=N M A(\delta, m, n)=\frac{1}{1-p(\delta, m, n)}
$$

Dado que o número médio de amostras (NMA) até identificar um ponto fora dos limites depende do erro de estimativa da média $\mu_{0}$, usa-se então a esperança matemática de NMA, chamado de Média da NMA (MNMA) para avaliar o desempenho dos gráficos de controle, dado que o processo é considerado fora de controle quando a média amostral cair na região M1. Assim, o NMA, conforme Saleh et al (2016), é calculado sobre todas as possíveis estimativas da fase I, e abordada na literatura como a média do NMA (MNMA). Portanto, a probabilidade de um ponto no gráfico cair na região $M 1$ é $P(\bar{x} \in M 1)=1-[P(\bar{x} \in M 0)+P(\bar{x} \in I)]$. Quando a 
média é estimada, o número total de parâmetros envolvidos no cálculo do MNMA é $\Omega=\left\{K_{1}, K_{2}, L_{1}, L_{2}, \delta, m, n\right\}$. Para o caso da média conhecida, o total de parâmetros do modelo é $\Omega=\left\{K_{1}, K_{2}, L_{1}, L_{2}, \delta, n\right\}$.

Assim, o MNMA para uma média conhecida é dado por:

$$
\operatorname{MNMA}\left(K_{1}, K_{2}, L_{1}, L_{2}, \delta, n\right)=\frac{1}{1-p\left(K_{1}, K_{2}, L_{1}, L_{2}, \delta_{,} n\right)}
$$

Quando a média é desconhecida, a medida incondicional do MNMA é dada por:

$$
M N M A=A R L\left(K_{1}, K_{2}, L_{1}, L_{2}, \delta, m, n\right)=\int_{-\infty}^{\infty} \frac{1}{[1-p(\delta, m, n)]} \varphi(w) d w
$$

Onde $\varphi$ denota a função distribuição de probabilidade $f(p) N(0,1)$ da variável aleatória.

Normalmente, utiliza-se o NMA como parâmetro de desempenho dos gráficos de controle estatísticos. Porém, quando os parâmetros estatísticos são desconhecidos, tal como a média e o desvio padrão populacional, e os mesmos são estimados na fase I da implantação dos gráficos estatísticos, os NMAs até detectarem um ponto fora de controle dependem da estimativa dos parâmetros. Chakraborti (2000) mostrou por meio de simulação que o desempenho dos gráficos de controle em termos do NMA é inferior ao desempenho quando os parâmetros são conhecidos. Jensen et al (2006) desenvolveram uma ampla revisão dos efeitos da estimativa dos parâmetros estatísticos. Do mesmo modo, Eppreacht et al (2015) e Saleh et al (2016) discutem novas propostas de mudanças nos gráficos de Shewhart para melhorá-los em termos do NMA. Esses autores propõem novos métodos de análise do desempenho dos gráficos de controle e formulam novos métodos de cálculo dos limites de controle.

\subsection{Design econômico para gráficos de controle com parâmetros variados}

No design estatístico, os parâmetros de design são escolhidos com base em considerações estatísticas, tais como probabilidades de erro do tipo I e tipo II. No design econômico, a escolha dos parâmetros de design é baseada numa função custo (DE MAGALHÃES; EPPRECHT; COSTA, 2001).

O design econômico de cartas tipo $\bar{X}$ com tamanhos de amostras variáveis foi estudado por Flaig (1991) e Park e Reynolds (1994). Estes últimos propuseram um modelo econômico para cartas tipo $\bar{X}$ com variação do tamanho da amostra quando Revista Produção Online. Florianópolis, SC, v.17, n. 3, p. 883-908, 2017. 
o processo está sujeito à ocorrência de várias causas atribuíveis. Das e Jain (1997) propuseram uma nova generalização de políticas de variação de intervalo amostral para cartas tipo $\bar{X}$ na qual os intervalos amostrais são tratados como variáveis aleatórias e os tamanhos amostrais são considerados uma função de intervalos amostrais. De Magalhães, Epprecht e Costa (2001) propuseram um modelo econômico para cartas de controle tipo $\bar{X}$ quando todos os parâmetros de design são variáveis. Os parâmetros de design são permitidos variar em tempo real com base na informação amostral. Um modelo foi desenvolvido, fornecendo uma função de custo que representa o custo por unidade de tempo para controlar a qualidade de um processo por meio de pV. Costa (1999) considerou o design estatístico de pV para cartas tipo $\bar{X}$ e mostrou que melhorias consideráveis de desempenho podem ser atingidas através de outras cartas de controle $\bar{X}$. Este estudo motivou De Magalhães, Epprecht e Costa (2001) a considerarem um modelo econômico para cartas tipo $\bar{X}$ tendo todos os parâmetros variáveis.

\section{MÉTODO DE PESQUISA}

Quanto ao método de pesquisa o presente trabalho enquadra-se como modelagem matemática, com resultados obtidos por métodos numéricos. De acordo com Nakano (2010, p.64), a modelagem trata-se do "uso de técnicas matemáticas para descrever o funcionamento de um sistema ou de parte de um sistema produtivo". Com base na finalidade, os objetivos são descritivos, e quanto aos procedimentos técnicos a pesquisa é bibliográfica.

As análises numéricas foram realizadas por meio do software Maple ${ }^{\circ}$ (no anexo A é evidenciado o programa desenvolvido para a simulação) e os resultados se encontram nos anexos B, C e D. Estes resultados apresentam os MNMAs do processo para avaliação do desempenho do gráfico de controle.

Tais resultados consideraram a estrutura apresentada na Figura 2. Assim, a primeira região (M0) é aquela no qual o processo assume estar em estado de controle, caso a estatística amostral caia nessa região. A região I é uma região de indiferença, isto é, caso uma estatística amostral caia nesta região, toma-se então mais uma amostra de mesmo tamanho. Repetida a amostragem, e a estatística amostral caia novamente na região I, outra amostra é extraída e deve-se então optar 
entre interromper o processo ou continuar operando. Quando o processo está sob controle, $\delta=0$, o desempenho dos gráficos é avaliado pela esperança matemática do número médio de amostra até detectar um ponto fora de controle e pelo tamanho médio da amostra. Por último a região M1 é definida pelos pontos que caírem acima do $\mathrm{LSC}_{2}$ e abaixo do $\mathrm{LIC}_{2}$.

De modo paralelo foi realizado um framework no intuito de auxiliar na construção de um design de gráficos de controle com limites assimétricos expandindo assim o conceito da abordagem de $\mathrm{pV}$.

\section{ANÁLISE DE DESEMPENHO DO GRÁFICO PROPOSTO COM PARÂMETROS VARIADOS (pV)}

Para a análise de desempenho dos pV foram utilizados os intervalos descritos no Quadro 2.

\begin{tabular}{|c|c|}
\hline Parâmetros & Intervalos \\
\hline$\delta$ & $0,00-0,25-0,50-0,75-1,00$ \\
\hline $\mathrm{K}_{1} ; \mathrm{K}_{2}$ & $0,00-0,25-0,50-0,75-1,00$ \\
\hline $\mathrm{L}_{1} ; \mathrm{L}_{2}$ & $2,00-2,25-2,50-2,75-3,00-3,25$ \\
\hline
\end{tabular}

Os parâmetros, tamanho e número de amostras, foram considerados como $\mathrm{n}=5$ e $\mathrm{m}=25$, respectivamente. Sendo que os valores do NMA para os parâmetros analisados foram estimados por meio do software de Maple ${ }^{\circledR}$, e encontram-se nos anexos B, C e D.

A Figura 3 demonstra o framework proposto e apresenta a sistemática do plano de amostragem para os gráficos de controle conforme os passos descritos a seguir:

- Inicia-se o procedimento tomando uma amostra de tamanho $n$ e calculase a estatística de teste (em outras palavras, calcula-se a estimativa do parâmetro estatístico monitorado, no presente caso, a média amostral): se a estatística da média amostral cair na região M0, aceita-se o processo como estável e dentro dos limites de controle e repete-se a amostragem em intervalos de tempo $(\Delta t)$ predeterminado; 
- Caso a estatística da média amostral caia na região $M 1$, para-se o processo e busca-se uma ou mais causas especiais (CE). Encontrada a causa especial e realizada as ações corretivas e efetividades voltam-se ao procedimento inicial de amostragem normal do processo;

- Se a estatística cair na região indiferente (Região I, da Figura 2), toma-se uma nova amostra de tamanho $n$. Se a nova estatística da média amostral situar-se na região MO se aceita o processo como adequado, e retorna-se ao procedimento normal. Caso a estatística da média amostral caia na região M1, para-se o processo e busca-se uma ou mais causas especiais;

- Caso a estatística da média amostral não se situe nas regiões M0 e M1, o procedimento indica que se tome uma terceira amostra. Caso esta amostra caia fora da região M0, para-se o processo e buscam-se as causas especiais, caso contrário retorna-se ao início do procedimento.

O framework proposto auxilia na construção de um design de gráficos com limites variáveis, podendo ser assimétricos, expandindo assim o conceito da abordagem de pV. No exemplo mostrado na Figura 3 são considerados os parâmetros $L_{1}=L_{2}=1, K_{1}=K_{2}=2, n=5$ e $\delta=0$. Os cálculos das probabilidades foram omitidos por simplificação, mas podem-se calcular as probabilidades para cada nó de decisão.

Para o exemplo de aplicação com os parâmetros acima, o NMA será de 319,7 (o usual é 370,4), ou seja, um número de amostras plotadas até a ocorrência de um sinal de parada considerada inadequada. Nessa linha de pensamento, se obteve por métodos numéricos a combinação de parâmetros com $\delta=0$, variando-se $K_{1}, K_{2}, L_{1} \mathrm{e}$ $L_{2}$ para número de amostras igual a 25 , cujo NMA deva ser o mesmo valor $(319,7)$. Os dados presentes no Quadro 3 indicam os valores das constantes usadas para a estimativa dos limites de controle do gráfico proposto. Estes dados foram extraídos do anexo B e obtidos por meio das equações mostradas na seção 2.2. 
Figura 3 - Framework proposto

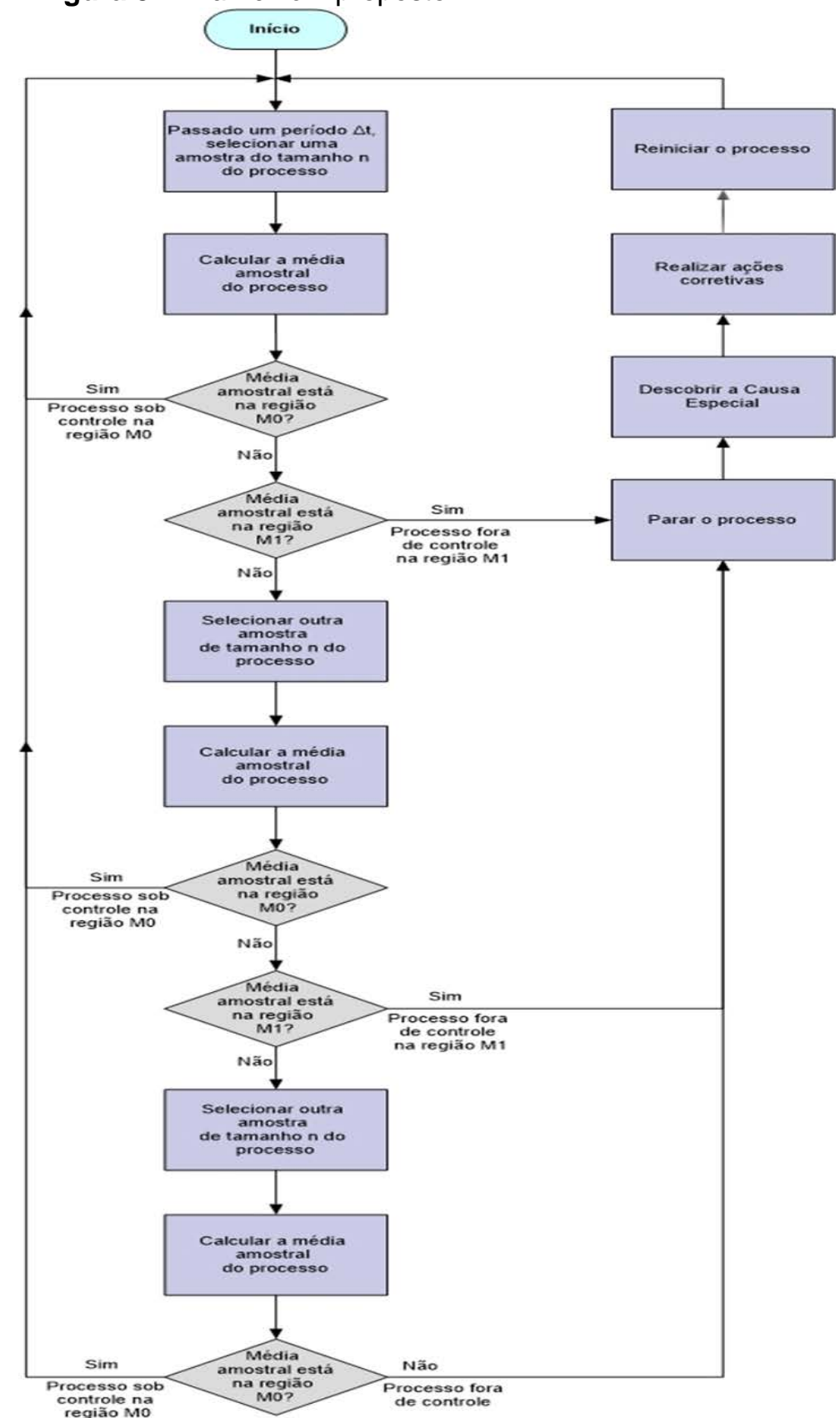

Fonte: Elaborado pelos autores (2017).

Quadro 3 - Combinação de parâmetros $\left(K_{1}, K_{2}, L_{1}, L_{2}\right)$ para $A R L=319,7$

$\begin{array}{llllllllllllllllllllllllll}\text { K1 } & 2,00 & 2,00 & 2,00 & 2,00 & 2,00 & 2,25 & 2,25 & 2,25 & 2,25 & 2,25 & 2,50 & 2,50 & 2,50 & 2,50 & 2,50 & 2,75 & 2,75 & 2,75 & 2,75 & 2,75 & 3,00 & 3,00 & 3,00 & 3,00 & 3,00\end{array}$

$\begin{array}{llllllllllllllllllllllllllll}\text { K2 } & 2,00 & 2,25 & 2,50 & 2,75 & 3,00 & 2,00 & 2,25 & 2,50 & 2,75 & 3,00 & 2,00 & 2,25 & 2,50 & 2,75 & 3,00 & 2,00 & 2,25 & 2,50 & 2,75 & 3,00 & 2,00 & 2,25 & 2,50 & 2,75 & 3,00\end{array}$

$\begin{array}{llllllllllllllllllllllllll}\text { L1 } & 1,00 & 1,00 & 1,00 & 1,00 & 1,00 & 0,75 & 0,75 & 0,75 & 0,75 & 0,75 & 0,50 & 0,50 & 0,50 & 0,50 & 0,50 & 0,25 & 0,25 & 0,25 & 0,25 & 0,25 & 0,00 & 0,00 & 0,00 & 0,00 & 0,00\end{array}$

$\begin{array}{lllllllllllllllllllllllllllll}\text { L2 } & 1,00 & 0,75 & 0,50 & 0,25 & 0,00 & 1,00 & 0,75 & 0,50 & 0,25 & 0,00 & 1,00 & 0,75 & 0,50 & 0,25 & 0,00 & 1,00 & 0,75 & 0,50 & 0,25 & 0,00 & 1,00 & 0,75 & 0,50 & 0,25 & 0,00\end{array}$

Fonte: Maple ${ }^{\circledR}(2016)$

A proposta apresentada pode ser aplicada em algumas situações práticas. Montgomery e Runger (2009), por exemplo, sugerem o uso de gráficos de controle

Revista Produção Online. Florianópolis, SC, v.17, n. 3, p. 883-908, 2017. 
modificados em situações onde a variabilidade natural de dispersão do processo é consideravelmente menor que a tolerância dos limites de especificação, nos casos onde os índices de capacidade de processo, $C_{p}$ (capacidade de processo) e $C_{p k}$ (índice de capacidade de processo), são muito maiores que 1. Woodall (1985) sobre isso acrescenta aos gráficos tradicionais de Shewhart limites de controle adicionais, de modo a reduzir o erro tipo I e consequentemente paradas desnecessárias do processo. Um exemplo prático de processos com altos índices de capacidade são procedimentos de estampagem de peças metálicas utilizadas em como componentes de sistemas de freios para automóveis, utilitários e caminhões.

O Manual do AIAG (1991) menciona situações como essas e métodos de controle alternativos. O livro Quality Control Handbook, de Juran e Gryna (1981), sobre o assunto, indicada o controle especial em processos com dominância de setup e pequena variabilidade em relação às tolerâncias de engenharia. Considerando a proposta de combinar limites assimétricos com amostragem múltipla, cabe destacar que são comuns em componentes automotivos especificações unilaterais (por exemplo, a concentricidade do diâmetro externo da rosca da conexão entre servo-freio e a mangueira de alimentação do fluído de freio, é uma característica crítica funcional do produto e assimétrica). De tal modo recomenda-se o uso de limites assimétricos em casos como o exemplo citado.

\section{CONSIDERAÇÕES FINAIS}

O desenvolvimento e utilização das técnicas e métodos estatísticos para a análise e solução de problemas passaram a ganhar importância no campo industrial, destacando a ferramenta CEP como eficiente para controle dos processos produtivos. Com a utilização do CEP é possível aproveitar melhor os equipamentos e máquinas, a mão-de-obra e os demais recursos, o que permite desenvolver ação gerencial para melhorias da qualidade e produtividade.

O presente artigo apresenta uma nova proposta de construção de gráficos de controle mais econômicos e robustos, utilizando-se pV para o cálculo dos limites de controle com três regiões distintas. O desempenho dos gráficos foi avaliado pela esperança matemática do número médio de amostra pelo cálculo do MNMA por meio de análises numéricas para auxiliar na análise crítica da proposta. A 
possibilidade de reamostragem do processo dependendo da região que a estatística amostral caia apresenta uma contribuição ao tema de construção de gráficos de controle com parâmetros variados (pV), considerando que os parâmetros estatísticos sejam desconhecidos.

Por ser um tema relativamente novo, pesquisas futuras com a utilização de métodos empíricos podem apresentar contribuições importantes ao tema. Análises de custos não foram consideradas neste trabalho, mas podem ser relevantes para comparações com outros métodos pV presentes na literatura.

\section{REFERÊNCIAS}

A.I.A.G. Fundamental Statistical Process Control. Detroit, MI: A.I.A.G, 1991.

AVINADAV, T.; PERLMAN, Y.; CHENG, T. C. E. Economic design of control charts for monitoring batch manufacturing processes. International Journal of Computer Integrated Manufacturing, v. 29, n. 2, p. 212-221, 2016. http://dx.doi.org/10.1080/0951192X.2015.1030699

CASTAGLIOLA, P.; CELANO, G.; CHEN, G. The Exact Run Length Distribution and Design of the S2 Chart When the in-Control Variance Is Estimated. International Journal of Reliability, Quality and Safety Engineering, v. 16, n. 1, p. 23-38, 2009. https://doi.org/10.1142/S0218539309003277

CASTAGLIOLA, P.; CELANO, G.; FICHERA, S. Comparison of the X-bar Chart and the $t$ Chart When the Parameters are Estimated. An International Journal Quality Technology \& Quantitative Management, v. 10, n. 1, p. 1-16, 2013.

http://dx.doi.org/10.1080/16843703.2013.11673305

CASTAGLIOLA, P.; MARAVELAKIS, P. E. A CUSUM control chart for monitoring the variance when parameters are estimated. Journal of Statistical Planning and Inference, v. 141, n. 4, p. 1463-1478, 2011. https://doi.org/10.1016/j.jspi.2010.10.013

CHAKRABORTI, S. Run length, average run length and false alarm rate of Shewhart X-bar chart: exact derivations by conditioning. Communications in Statistics, v.28, n.7, p. 61-81, 2000. http://dx.doi.org/10.1080/03610910008813602

CHEN, F. L.; YEH, L. L. Economic statistical design of $x$-bar control charts for correlated data and Gamma failure mechanism with genetic algorithm, 40th International Conference on Computers and Industrial Engineering: Soft Computing Techniques for Advanced Manufacturing and Service Systems, CIE40, 25-28 july 2010. https://doi.org/10.1109/ICCIE.2010.5668330

$\mathrm{CHEN}, \mathrm{H}$. Joint determination of economic manufacturing quantity and control limits of x-bar control chart. 15th ISSAT International Conference on Realiability and Quality in Design, San Francisco, CA, United Stades, 2009.

CHEN, H.; KUO, W.-L. Comparisons of the symmetric and asymmetric control limits for Revista Produção Online. Florianópolis, SC, v.17, n. 3, p. 883-908, 2017. 
x-bar charts. IEEM International Conference on Industrial Engineering and Engineering Menagement, 2007. https://doi.org/10.1109/IEEM.2007.4419418

CHEN, H.; KUO, W.-L. Comparisons of the Symmetric and Asymmetric control limits for $\mathrm{X}-$ bar and R charts. Computers \& Industrial Engineering, v. 59, n. 4, p. 903-910, 2010. https://doi.org/10.1016/i.cie.2010.08.021

COSTA, A. F. B. Vssi X-bar charts with sampling at fixed times. Communications in Statistics - Theory and Methods, v. 27, n. 11, p. 2853-2869, 1998a. http://dx.doi.org/10.1080/03610929808832259

COSTA, A. F. B. Joint $X$-bar and $R$ charts with variable parameters. IIE Transactions, v. 30, n. 6, p. 505-414, 1998b. DOI: 10.1023/A\%3A1007555625827

COSTA, A. F. B. X-bar Charts with Variable Parameters. Journal of Quality Technology, v. 31, n. 4, 1999.

COSTA, A. F. B.; DE MAGALHÃES, M. S. Economic design of two-stage $x$-bar charts: The Markov chain approach. International Journal of Production Economics2, v. 95, n. 1, p. 9-20, 2005. https://doi.org/10.1016/j.ijpe.2003.10.024

DAS, T. K.; JAIN, V. An economic design model for (X)over-bar charts with random sampling policies. lie Transactions, v. 29, n. 6, p. 507-518, 1997.

http://dx.doi.org/10.1080/07408179708966357

DE MAGALHÃES, M. S.; EPPRECHT, E. K.; COSTA, A. F. B. Economic design of a Vp X bar chart. International Journal of Production Economics, v. 74, p. 191-200, 2001. https://doi.org/10.1016/S0925-5273(01)00126-8

DE MAGALHÃES, M. S.; MOURA NETO, F. D. Joint economic model for totally adaptive xbar and R charts. European Journal of Operational Research, v. 161, n. 1, p. 148-161, 2005. https://doi.org/10.1016/j.ejor.2003.08.033

EPPRECHT, E. K.; LOUREIRO, L. D.; CHAKRABORTI, S. Effect of the amount of Phase I data on the Phase II performance of $S^{2}$ and $S$ Control Charts. Journal of Quality Technology, v. 47, n.2, p. 139-155, 2015.

FERREIRA, E. B.; ELISEI JUNIOR, L.; MILITANI, M. V. B. Controle estatístico de processo no resfriamento de aves: um estudo de caso. Revista da Universidade Vale do Rio Verde, v. 9, n. 2, p. 119-128, 2011. http://dx.doi.org/10.5892/ruvrv.2011.92.119128

FLAIG, J. J. Adaptative Control Charts. In: KEATS, J. B.; MONTGOMERY, D. C. (Eds.). . Statistical process control in manufacturing. 1. ed. New York: Marcel Dekker Inc, 1991. p. 111-122.

JENSEN, W. A. et al. Effects of Parameter Estimation on Control Chart Properties ; A Literature Review. Journal of Quality Technology, v. 38, n. 4, p. 349-364, 2006.

JURAN, Joseph M.; GRYNA, Frank M.; BOU, José María Vallhonrat. Planificación y análisis de la calidad. Reverté, 1981.

KORZENOWSKI, A. L. Controle estatístico do processo aplicado a ambientes customizados. Tese de doutorado apresentada ao Programa de Pós-Graduação em Engenharia de Produção, Universidade Federal do Rio Grande do Sul, 2012. 
MARSHALL JUNIOR, I. et al. Gestão da Qualidade. 2. ed. Rio de Janeiro: Editora FGV, 2003.

MOBIN, M.; LI, Z.; KHORASKANI, M. M. Multi-Objective X-bar control chart design by integrating NSGA-II and data envelopment analysis. IIE Annual Conference and Expo 2015. Rensissance Nashville HotelNashville; United States: 2015.

MONTGOMERY, D. C. Introdução ao controle estatístico da qualidade. 4. ed. Rio de Janeiro: LTC, 2004.

MONTGOMERY, D. C.; RUNGER, G. C. Estatística aplicada e probabilidade para engenheiros. 4. ed., LTC, 2009. 463 p.

OPRIME, P. C.; GANGA, G. M. D. Análise do desempenho de gráfico de controle da variância com parâmetro estimado. XXXV Encontro Nacional de Engenharia de Produção. Anais...Fortaleza - CE: ENEGEP, 2015.

NAKANO, D. Métodos de Pesquisa Adotados na Engenharia de Produção e Gestão de Operações, In: MIGUEL, P, A, C, (org,), Metodologia de pesquisa em engenharia de produção e gestão de operações, Rio de Janeiro: Elsevier, 2010, p,63-72.

PARK, C.; CHOI, K. An economic design of control charts with VSS-Scheme. Presented at the joint Statistical Meetings of the American Statistical Association, Boston, 1992.

PARK, C.; REYNOLDS, M. R. Economic design of a variable sample size - chart.

Communications in Statistics - Simulation and Computation, v. 23, n. 2, p. 467-483, 1994. http://dx.doi.org/10.1080/03610919408813182

PEÑABAENA-NIEBLES, R. et al. Methodology for the implementation of an economic and/or statistical design for $x$-bar charts with variable parameters (VP). Dyna, v. 81, n. 184, p. 150157, 2014. http://dx.doi.org/10.15446/dyna.v81n184.35510

PRABHU, S. S.; MONTGOMERY, D. C.; RUNGER, G. C. A Combined Adaptive Sample Size and Sampling Interval X Control Scheme. Journal of Quality Technology, v. 26, n. 3, 1994.

REYNOLDS, M. R. et al. X-Bar Charts with Variable Sampling Intervals. Technometrics, v. 30, n. 2, p. 181-192, 1988. http://dx.doi.org/10.1080/00401706.1988.10488366

REYNOLDS, M. R.; AMIN, R. W.; ARNOLD, J. C. CUSUM Charts with Variable Sampling Intervals. Technometrics, v. 32, n. 4, p. 371-384, 1990.

http://dx.doi.org/10.1080/00401706.1990.10484721

SACCUCCI, M. S.; AMIN, R. W.; LUCAS, J. M. Exponentially weighted moving average control schemes with variable sampling intervals. Communications in Statistics Simulation and Computation, v. 21, n. 3, p. 627-657, 1992.

http://dx.doi.org/10.1080/03610919208813040

SAFAEI, A. S.; KAZEMZADEH, R. B.; GAN, H. Robust economic-statistical design of X-bar control chart. International Journal of Production Research, v. 53, n. 14, p. 4446-4458, 2015. http://dx.doi.org/10.1080/00207543.2015.1018449

SALEH, N. A.; ZWETSLOOT, I. M.; MAHMOUD, M. A.; WOODALL, W. H. CUSUM charts Revista Produção Online. Florianópolis, SC, v.17, n. 3, p. 883-908, 2017. 
with controlled conditional performance under estimated parameters. Quality Engineering, v. 28 , n. 4, p. 402-415, 2016. http://dx.doi.org/10.1080/08982112.2016.1144072

SCHOONHOVEN, M.; DOES, R. J. M. M. A Robust Standard Deviation Control Chart. Technometrics, v. 54, n. 1, p. 73-82, 2012. http://dx.doi.org/10.1080/00401706.2012.648869

VELJKOVIC, K.; ELFAGHIHE, H.; JEVREMOVIC, V. Economic Statistical Design of X Bar Control Chart for Non-Normal Symmetric Distribution of Quality Characteristic. Filomat, v. 29, n. 10, p. 2325-2338, 2015. http://dx.doi.org/10.2298/FIL1510325V

VRIES, A.; RENEAU, J. K. Application of statistical process control charts to monitor changes in animal production systems. Journal of Animal Scince, v. 88, n. 13, p. 11-24, 2010. http://dx.doi.org/10.2527/jas.2009-2622

WOODALL, W. H. The statistical design of quality control charts. The Statistician, v. 34, n. 2, p. 155-160, 1985. http://dx.doi.org/10.2307/2988154

YANG, Z. et al. On the Performance of Geometric Charts with Estimated Control Limits. Journal of Quality Technology, v. 34, n. 2, 2002.

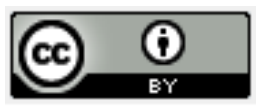

Artigo recebido em 07/02/2017 e aceito para publicação em 27/07/2017

DOI: http://dx.doi.org/10.14488/1676-1901.v17i3.2722 


\section{Anexo A - Software Maple ${ }^{\circledR}$}

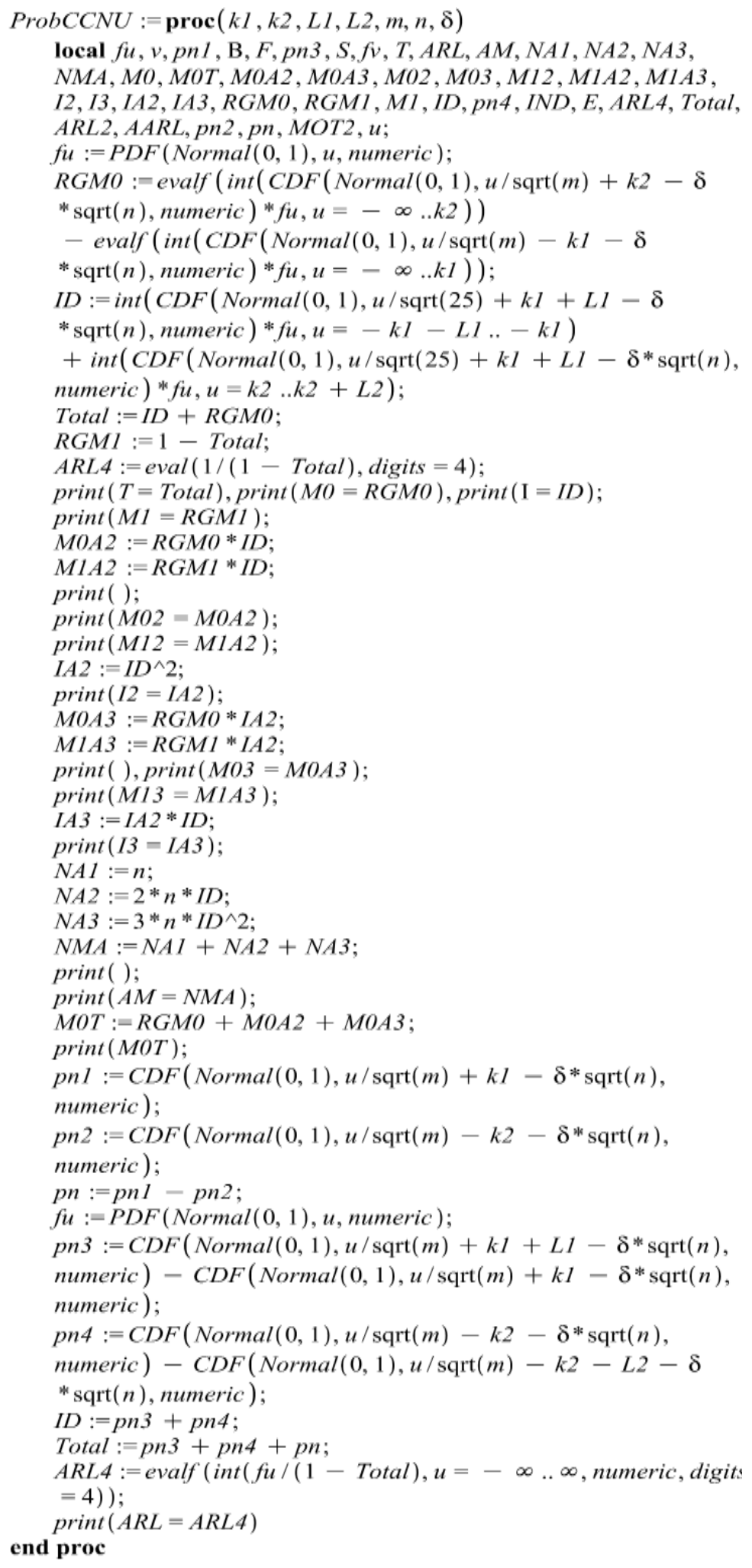


Anexo B - Valores de MNMA. $\delta=0$

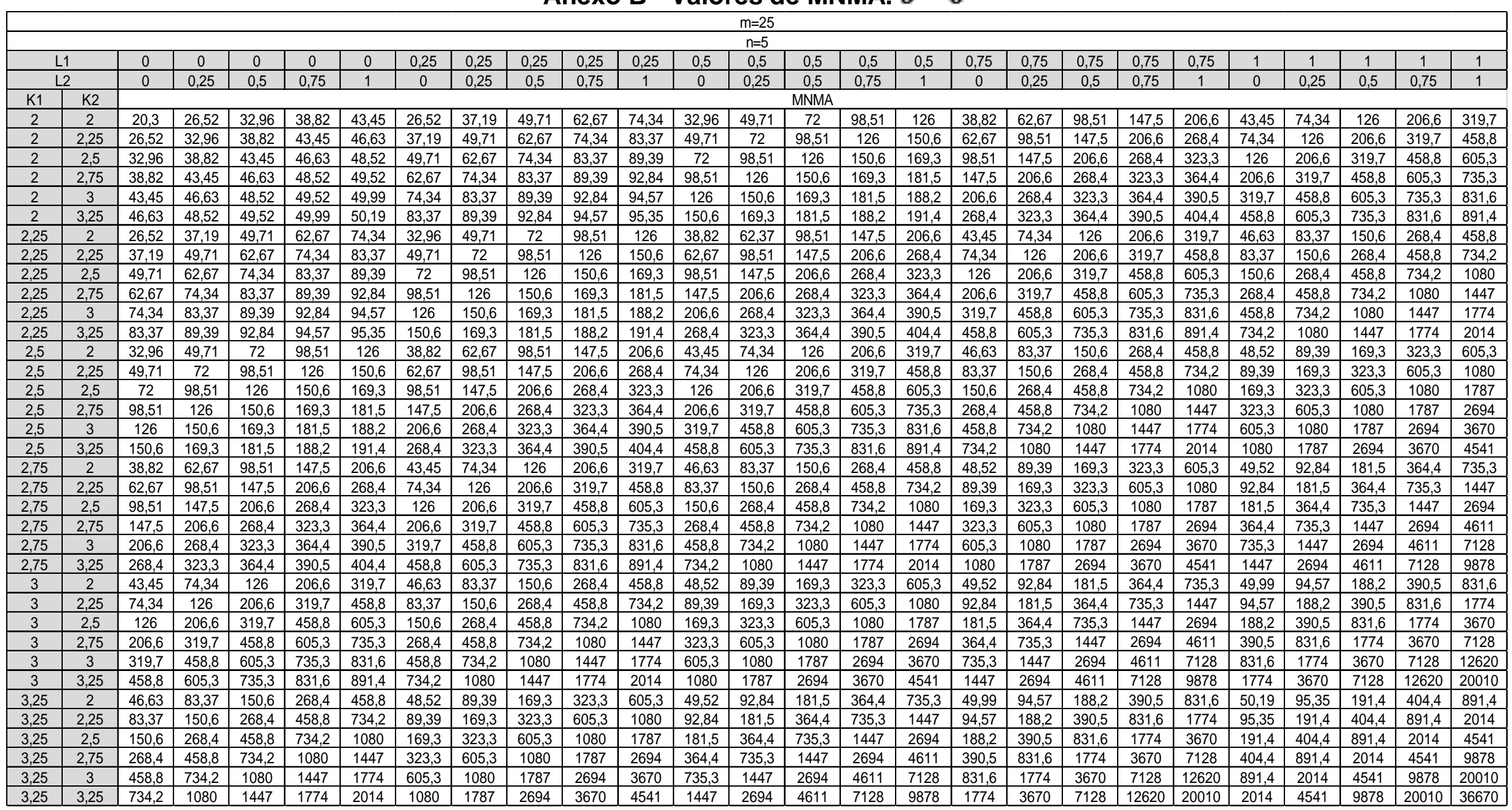

Revista Produção Online. Florianópolis, SC, v.17, n. 3, p. 883-908, 2017. 
Anexo C - Valores de MNMA. $\delta=0,25$

\begin{tabular}{|c|c|c|c|c|c|c|c|c|c|c|c|c|c|c|c|c|c|c|c|c|c|c|c|c|c|c|}
\hline \multicolumn{27}{|c|}{$m=25$} \\
\hline \multicolumn{27}{|c|}{$\mathrm{n}=5$} \\
\hline \multirow{2}{*}{\multicolumn{2}{|c|}{$\frac{\mathrm{L} 1}{\mathrm{~L} 2}$}} & 0 & 0 & 0 & 0 & 0 & 0.25 & 0.25 & 0.25 & 0.25 & 0.25 & 0.50 & 0.50 & 0.50 & 0.50 & 0.50 & 0.75 & 0.75 & 0.75 & 0.75 & 0.75 & 1 & 1 & 1 & 1 & 1 \\
\hline & & 0 & 0.25 & 0.50 & 0.75 & 1 & 0 & 0.25 & 0.5 & 0.75 & 1 & $\pi_{0}$ & 0.25 & 0.5 & 0.75 & 4 & 8 & 0.25 & 0.5 & 0.75 & 1 & 0 & 0.25 & 0.5 & 0.75 & 1 \\
\hline K1 & $\mathrm{K} 2$ & \multicolumn{25}{|c|}{ MNMA } \\
\hline 2 & 2 & 12.84 & 13.63 & 4.13 & 14.40 & 14.54 & 19.88 & 21.79 & 23.07 & 23.83 & 4.23 & 1.02 & 35.60 & 39.01 & 41.22 & 42.47 & 47.85 & 58.46 & 57.38 & 73.86 & 77.90 & 71.43 & 94.32 & 116.5 & 134.9 & 148.0 \\
\hline 2 & 2,25 & 13.63 & 14.13 & 14.40 & 14.54 & 14.60 & 21.79 & 23.07 & 23.83 & 24.23 & 24.42 & 35.60 & 39.01 & 41.22 & 42.47 & 43.10 & 58.46 & 67.38 & 73.86 & 77.90 & 80.10 & 94.32 & 116.5 & 134.9 & 148.0 & 155.9 \\
\hline 2 & 2,5 & 14.13 & 14.40 & 14.54 & 14.60 & 14.63 & 23.07 & 23.83 & 24.23 & 24.42 & 24.5 & 39.01 & 41.22 & 42.47 & 3.10 & 43.39 & 67.38 & 73.86 & 77.90 & 80.10 & 81.15 & 116.5 & 134.9 & 148.0 & 155.9 & 160.0 \\
\hline 2 & 2,75 & 14.40 & 14.54 & 4.60 & 14.63 & 14.64 & 23.83 & 24.23 & 24.42 & 24.51 & 24.5 & 41.22 & 42.47 & 43.10 & 43.39 & 43.50 & 73.86 & 77.90 & 80.10 & 81.15 & 81.60 & 134.9 & 148.0 & 155.9 & 160.0 & 161.9 \\
\hline 2 & 3 & 14.54 & 14.60 & 14.63 & 14.64 & 14.64 & 24.23 & 24.42 & 24.51 & 24.54 & 24.55 & 42.47 & 43.10 & 43.39 & 3.50 & 43.55 & 77.90 & 80.10 & 81.15 & 81.60 & 81.78 & 48.0 & 155.9 & 160.0 & 61.9 & 162.7 \\
\hline 2 & 3,25 & 14.60 & 14.63 & 14.64 & 14.64 & 14.65 & 24.42 & 24.51 & 24.54 & 24.55 & 24.56 & 43.10 & 43.39 & 43.50 & 3.55 & 43.57 & 80.10 & 81.15 & 81.60 & 81.78 & 81.85 & 155.9 & 160.0 & 161.9 & 162.7 & 163.0 \\
\hline 2,25 & 2 & 19.88 & 21.79 & 23.07 & 23.83 & 24.23 & 31.02 & 35.60 & 39.01 & 41.22 & 42.47 & 47.85 & 58.46 & 67.38 & 73.86 & 77.90 & 71.43 & 94.32 & 116.5 & 134.9 & 148.0 & 101.2 & 146.1 & 196.8 & 245.8 & 286.2 \\
\hline 2,25 & 2.25 & 21.79 & 23.07 & 23.83 & 24.23 & 24.42 & 35.60 & 39.01 & 41.22 & 42.47 & 43.10 & 58.46 & 67.38 & 73.86 & 77.90 & 80.10 & 94.32 & 116.5 & 134.9 & 148.0 & 155.9 & 146.1 & 196.8 & 245.8 & 286.2 & 314.2 \\
\hline 2,25 & 2.5 & 23.07 & 23.83 & 24.23 & 24.42 & 24.51 & 39.01 & 41.22 & 42.47 & 43.10 & 43.39 & 67.38 & $\begin{array}{l}73.86 \\
\end{array}$ & 77.90 & 80.10 & 81.15 & 116.5 & 134.9 & 148.0 & 155.9 & 160.0 & 196.8 & 245.8 & 286.2 & 314.2 & 330.7 \\
\hline 2,25 & 2.75 & 23.83 & 24.23 & 24.42 & 24.51 & 24.54 & 41.22 & 42.47 & 43.10 & 43.39 & 43.50 & 73.86 & 77.90 & 80.10 & 81.15 & 81.60 & 134.9 & 148.0 & 155.9 & 160.0 & 161.9 & 245.8 & 286.2 & 314.2 & 330.7 & 339.0 \\
\hline 2,25 & 3 & 24.23 & 24.42 & 24.51 & 24.54 & 24.55 & 42.47 & 43.10 & 43.39 & 43.50 & 43.55 & 77.90 & 80.10 & 81.15 & 81.60 & 81.78 & 148.0 & 155.9 & 160.0 & 161.9 & 162.7 & 286.2 & 314.2 & 330.7 & 339.0 & 342.6 \\
\hline 2.25 & 3.25 & 24.42 & 24.51 & 24.54 & 24.55 & 24.56 & 43.10 & 43.39 & 43.50 & 43.55 & 43.57 & 80.10 & 81.15 & 81.60 & 81.78 & 81.85 & 155.9 & 160.0 & 161.9 & 162.7 & 163.0 & 314.2 & 330.7 & 339.0 & 342.6 & 344.0 \\
\hline 2,5 & 2 & 31.02 & 35.60 & 39.01 & 41.22 & 42.47 & 47.85 & 58.46 & 67.38 & 73.86 & 77.90 & 71.43 & 94.32 & 116.5 & 134.9 & 134.9 & 101.2 & 146.1 & 196.8 & 245.8 & 286.2 & 134.4 & 213.0 & 316.5 & 434.7 & 549.5 \\
\hline 2,5 & 2.25 & 35.60 & 39.01 & 41.22 & 42.47 & 43.10 & 58.46 & $\begin{array}{l}67.38 \\
\end{array}$ & 73.86 & 77.90 & 80.10 & 94.32 & 116.5 & $\begin{array}{ll}134.9 \\
\end{array}$ & 134.9 & 155.9 & 146.1 & 196.8 & 245.8 & 286.2 & 314.2 & 213.0 & 316.5 & 434.7 & 549.5 & 643.2 \\
\hline 2,5 & 2.5 & 39.01 & 41.22 & 42.47 & 43.10 & 43.39 & 67.38 & 73.86 & 77.90 & 80.10 & 81.15 & 116 & 134.9 & & 155.9 & 160.0 & 196.8 & 245.8 & 286.2 & 314.2 & 330.7 & 316.5 & 434.7 & 549.5 & 643.2 & 707.3 \\
\hline 2.5 & 2,75 & 41.22 & 42.47 & 43.10 & 43.39 & 43.50 & 73.86 & 77.90 & 80.10 & 81.15 & 81.60 & 134 & 134.9 & 155.9 & 160.0 & 161.9 & 245.8 & 286.2 & 314.2 & 330.7 & 339.0 & 434.7 & 549.5 & 643.2 & 707.3 & 744.1 \\
\hline 2.5 & 3 & 42.47 & 43.10 & 43.39 & 43.50 & 43.55 & 77.90 & 80.10 & 81.15 & 81.60 & 81.7 & & 155.9 & & & 162.7 & & 314. & & 339.0 & 342.6 & 5 & 643.2 & 707.3 & 744.1 & 761.9 \\
\hline 2.5 & 3,25 & 43.10 & 43.39 & 43.50 & 43.55 & 43.57 & 80.10 & 81.15 & 81.60 & 81.78 & 81.85 & 155 & 160.0 & 161.9 & 162.7 & 163.0 & 314.2 & 330.7 & 339.0 & 342.6 & 344.0 & 643.2 & 707.3 & 744.1 & 761.9 & 769.5 \\
\hline 2.75 & 2 & 47.85 & 58.46 & 67.38 & 73.86 & 77.90 & 71.43 & 94.32 & 116.5 & 134.9 & 148.0 & 101 & 146.1 & 196.8 & 245.8 & 286.2 & 134.4 & 213.0 & 316.5 & 434.7 & 549.5 & 166.2 & 288.2 & $\begin{array}{l}474.7 \\
\end{array}$ & 726.1 & 1017. \\
\hline 2.75 & 2.25 & 58.46 & 67.38 & 73.86 & 77.90 & 80.10 & 94.32 & 116.5 & 134.9 & 148.0 & 155.9 & 146 & $\begin{array}{l}196.8 \\
\end{array}$ & 245.8 & 286.2 & 314.2 & 213.0 & 316.5 & $\begin{array}{l}434.7 \\
\end{array}$ & 549.5 & 643.2 & 288.2 & 474.7 & 726.1 & 1017. & 1302. \\
\hline 2.75 & 2.5 & $\begin{array}{r}67.38 \\
\end{array}$ & 73.86 & 77.90 & 80.10 & 81.15 & 116.5 & 134.9 & 148.0 & 155.9 & 160.0 & 196.8 & 245.8 & 286.2 & 314.2 & 330.7 & 316.5 & 434.7 & 549.5 & 643.2 & 707.3 & 474.7 & 726.1 & 1017. & 1302. & 1534. \\
\hline 2.75 & 2.75 & $\begin{array}{l}73.86 \\
\end{array}$ & 77.90 & 80.10 & 81.15 & 81.60 & 134.9 & 148.0 & 155.9 & 160.0 & 161.9 & 245 & 286.2 & $\begin{array}{l}14.2 \\
\end{array}$ & 330.7 & 339.0 & 434.7 & 549.5 & 643.2 & 707.3 & 744.1 & 726.1 & 1017. & 1302. & 1534. & 1690. \\
\hline 2.75 & 3 & 77.90 & 80.10 & 81.15 & 81.60 & 81.78 & 148.0 & 155.9 & 160.0 & 161.9 & 162.7 & 286.2 & 314.2 & 330.7 & 339.0 & 342.6 & 549.5 & 643.2 & 707.3 & 744.1 & 761.9 & 1017. & 1302. & 1534. & 1690. & 1777. \\
\hline 2.75 & 3.25 & 80.10 & 81.15 & 81.60 & 81.78 & 81.85 & 155.9 & 160.0 & 161.9 & 162.7 & 163.0 & 31 & 330.7 & & 342.6 & 344.0 & & 707.3 & 744.1 & 761.9 & 769.5 & 1302. & 1534. & 1690. & 1777. & 1819. \\
\hline 3 & 2 & 71.43 & 94.32 & 116.5 & 134.9 & 148.0 & 101.2 & 146.1 & 196.8 & 245.8 & 286.2 & 134 & 213.0 & 316.5 & 434.7 & 549.5 & 166.2 & 288.2 & 474.7 & 726.1 & 1017. & 192.3 & 360.5 & 654.9 & 1121. & 1765. \\
\hline 3 & 2.25 & 94.32 & 116.5 & 134.9 & 148.0 & 155.9 & 146.1 & 196.8 & 245.8 & 286.2 & 314.2 & 21 & 316.5 & 434.7 & 549.5 & 643.2 & 288.2 & 474.7 & 726.1 & 1017. & 1302. & 360.5 & 654.9 & 1121. & 1765. & 2525. \\
\hline 3 & 2.5 & 116.5 & 134.9 & 148.0 & 155.9 & 160.0 & 196.8 & 245.8 & 286.2 & 314.2 & 314.2 & 31 & 434.7 & 549.5 & 643.2 & 707.3 & 474.7 & 726.1 & 1017. & 1302. & 1534. & 654.9 & 1121. & 1765. & 2525. & 3274. \\
\hline 3 & 2.75 & 134.9 & 148.0 & 155.9 & 160.0 & 161.9 & 245.8 & 286.2 & 314.2 & 314.2 & 339. & 434.7 & 549.5 & 643.2 & 707.3 & 744.1 & 726.1 & 1017. & 1302. & 1534. & 1690. & 1121. & 1765. & 2525. & 3274. & 3881. \\
\hline 3 & 3 & 148.0 & 155.9 & 160.0 & 161.9 & 162.7 & 286.2 & 314.2 & 314.2 & 339.0 & 342.6 & 549.5 & 643.2 & 707.3 & 744.1 & 761.9 & 1017. & 1302. & 1534. & 1690. & 1777. & 1765. & 2525. & 3274. & 3881. & 4287. \\
\hline 3 & 3.25 & 155.9 & 160.0 & 161.9 & 162.7 & 163.0 & 314.2 & 314.2 & 339.0 & 342.6 & 344. & 643.2 & 707.3 & 744.1 & 761.9 & 769.5 & 1302. & 1534. & 1690. & 1777. & 1819. & 2525 . & 3274. & 3881. & 4287. & 4511. \\
\hline 3.25 & 2 & 101.2 & 146.1 & 196.8 & 245.8 & 286.2 & 134.4 & 213.0 & 316.5 & 434.7 & 549.5 & 166 & 288.2 & $\begin{array}{l}474.7 \\
\end{array}$ & 726.1 & 1017. & 192.3 & 360.5 & 654.9 & 1121. & 1765. & 210.4 & 419.2 & 828.8 & 1577. & 2805. \\
\hline 3.25 & 2.25 & 146.1 & 196.8 & 245.8 & 286.2 & 314.2 & 213.0 & 316.5 & 434.7 & 549.5 & 643.2 & 288.2 & 474.7 & 726.1 & 1017. & 1302. & 360.5 & 654.9 & 1121. & 1765. & 2525 . & 419.2 & 828.8 & 1577. & 2805 . & 4551. \\
\hline 3.25 & 2.5 & 196.8 & 245.8 & 286.2 & 314.2 & 330 & 316 & 434.7 & 549.5 & 643 & 707 & 474 & 726.1 & 101 & 1302 & 1534 & 654.9 & 1121 & 176 & 2525 & 3274. & 828.8 & 15 & 2805. & 4551. & 6649. \\
\hline 3.25 & 2.75 & 245.8 & 286.2 & 314.2 & 330.7 & 339.0 & 434.7 & 549.5 & 643.2 & 707.3 & 744.1 & 726 & 1017. & 1302. & 1534. & 1690. & 1121. & 1765. & 2525. & 3274. & 3881. & 1577. & 2805. & 4551. & 6649. & 8734. \\
\hline 3.25 & 3 & 286.2 & 314.2 & 330.7 & 339.0 & 342.6 & 549.5 & 643.2 & 707.3 & 744.1 & 761. & 1017. & 1302. & 1534. & 1690. & 1777 & 1765. & 2525. & 3274. & 3881. & 4287. & 2805. & 4551. & 6649. & 8734. & 10430. \\
\hline 3.25 & 3.25 & 314.2 & 330.7 & 339.0 & 342.6 & 344.0 & 643.2 & 707.3 & 744.1 & 761.9 & 769.5 & 1302. & 1534. & 1690. & 1777. & 1819. & 2525. & 3274 . & 3881. & 4287. & 4511. & 4551. & 6649. & 8834. & 10430. & 11560. \\
\hline
\end{tabular}

Revista Produção Online. Florianópolis, SC, v.17, n. 3, p. 883-908, 2017. 
Anexo D - Valores de MNMA. $\delta=0,5$

\begin{tabular}{|c|c|c|c|c|c|c|c|c|c|c|c|c|c|c|c|c|c|c|c|c|c|c|c|c|c|c|}
\hline \multicolumn{27}{|c|}{$\mathrm{m}=25$} \\
\hline \multicolumn{27}{|c|}{$n=5$} \\
\hline \multirow{2}{*}{\multicolumn{2}{|c|}{$\frac{\mathrm{L} 1}{\mathrm{~L} 2}$}} & 0 & 0 & 0 & 0 & 0 & 0.25 & 0.25 & 0.25 & 0.25 & 0.25 & 0.50 & 0.50 & 0.50 & 0.50 & 0.50 & 0.75 & 0.75 & 0.75 & 0.75 & 0.75 & 1 & 1 & 1 & 1 & 1 \\
\hline & & 0 & 0.25 & 0.50 & 0.75 & 1 & 0 & 0.25 & 0.5 & 0.75 & 1 & 0 & 0.25 & 0.5 & 0.75 & 1 & 0 & 0.25 & 0.5 & 0.75 & 1 & 0 & 0.25 & 0.5 & 0.75 & 1 \\
\hline $\mathrm{K} 1$ & $\mathrm{~K} 2$ & \multicolumn{25}{|c|}{ MNMA } \\
\hline 2 & 2 & 5.556 & 5.587 & 5.602 & .608 & 5.611 & 8.207 & 8.280 & 8.315 & 8.330 & 8.336 & 12.72 & 12.91 & 13.00 & 13.04 & 13.06 & 20.65 & 21.16 & 21.42 & 21.55 & 21.60 & 34.86 & 36.37 & 37.19 & 37.58 & 37.75 \\
\hline 2 & 2,25 & 587 & 5.602 & 5.608 & 611 & 5.612 & \begin{tabular}{|l}
8.280 \\
\end{tabular} & 8.315 & 8.330 & 8.336 & 8.339 & 12.91 & 13.00 & 13.04 & 13.06 & 13.07 & 21.16 & 21.42 & 21.55 & 21.60 & 21.62 & 36.37 & 37.19 & 37.58 & 37.75 & 37.82 \\
\hline 2 & 2,5 & 602 & 5.608 & 5.611 & 612 & 5.612 & \begin{tabular}{|l|l|}
8.315 \\
\end{tabular} & 8.330 & 8.336 & 8.339 & 8.340 & 13.00 & 13.04 & 13.06 & 13.07 & 13.07 & 21.42 & 21.55 & 21.60 & 21.62 & 21.63 & 37.19 & 37.58 & 37.75 & 37.82 & 37.85 \\
\hline 2 & 2,75 & 5.608 & 5.611 & 5.612 & .612 & 5.612 & \begin{tabular}{|l}
8.330 \\
\end{tabular} & 8.336 & 8.339 & 8.340 & 8.340 & 13.04 & 13.06 & 13.07 & 13.07 & 13.07 & 21.55 & 21.60 & 21.62 & 21.63 & 21.63 & 37.58 & 37.75 & 37.82 & 37.85 & 37.86 \\
\hline 2 & 3 & 611 & 5.612 & 5.612 & 5.612 & 5.612 & \begin{tabular}{|l}
8.336 \\
\end{tabular} & 8.339 & 8.340 & 8.340 & 8.340 & 13.06 & 13.07 & 13.07 & 13.07 & 13.07 & 21.60 & 21.62 & 21.63 & 21.63 & 21.63 & 37.75 & 37.82 & 37.85 & 37.86 & 37.86 \\
\hline 2 & 3,25 & 612 & 5.612 & 5.612 & 5.612 & 5.612 & \begin{tabular}{|l}
8.339 \\
\end{tabular} & 8.340 & 8.340 & 8.340 & 8.340 & 13.07 & 13.07 & 13.07 & 13.07 & 13.07 & 21.62 & 21.63 & 21.63 & 21.63 & 21.63 & 37.82 & 37.85 & 37.86 & 37.86 & 37.86 \\
\hline 2,25 & 2 & 207 & 8.280 & 8.315 & 8.330 & 8.336 & \begin{tabular}{|c|}
12.72 \\
\end{tabular} & 12.91 & 13.00 & 13.04 & 13.06 & 20.65 & 21.16 & 21.42 & 21.55 & 21.60 & 34.86 & 36.37 & 37.19 & 37.58 & 37.75 & 60.55 & 65.08 & 67.75 & 69.13 & 69.76 \\
\hline 2,25 & 2.25 & 8.280 & 8.315 & 8.330 & 8.336 & 8.339 & \begin{tabular}{|l|}
12.91 \\
\end{tabular} & 13.00 & 13.04 & 13.06 & 13.07 & 21.16 & 21.42 & 21.55 & 21.60 & 21.62 & 36.37 & 37.19 & 37.58 & 37.75 & 37.82 & 65.08 & 67.75 & 69.13 & 69.76 & 70.03 \\
\hline 2,25 & 2.5 & 8.315 & 8.330 & 8.336 & 8.339 & 8.340 & 13.00 & 13.04 & 13.06 & 13.07 & 13.07 & 21.42 & 21.55 & 21.60 & 21.62 & 21.63 & 37.19 & 37.58 & 37.75 & 37.82 & 37.85 & 67.75 & 69.13 & 69.76 & 70.03 & 70.13 \\
\hline 2,25 & 2.75 & 30 & 8.336 & 8.339 & 8.340 & 8.340 & 13.04 & 13.06 & 13.07 & 13.07 & 13.07 & 21.55 & 21.60 & 21.62 & 21.63 & 21.63 & 37.58 & 37.75 & 37.82 & 37.85 & 37.86 & 69.13 & 69.76 & 70.03 & 70.13 & 70.16 \\
\hline 2,25 & 3 & 336 & 8.339 & 8.340 & 8.340 & 8.340 & \begin{tabular}{|l}
13.06 \\
\end{tabular} & 13.07 & 13.07 & 13.07 & 13.07 & 21.60 & 21.62 & 21.63 & 21.63 & 21.63 & 37.75 & 37.82 & 37.85 & 37.86 & 37.86 & 69.76 & 70.03 & 70.13 & 70.16 & 70.18 \\
\hline 2.25 & 3.25 & 339 & 8.340 & 8.340 & 8.340 & 8.340 & \begin{tabular}{|l|l}
13.07 \\
\end{tabular} & 13.07 & 13.07 & 13.07 & 13.07 & 21.62 & 21.63 & 21.63 & 21.63 & 21.63 & 37.82 & 37.85 & 37.86 & 37.86 & 37.86 & 70.03 & 70.13 & 70.16 & 70.18 & 70.18 \\
\hline 2,5 & 2 & 12.72 & 12.91 & 13.00 & 13.04 & 13.06 & 20.65 & 21.16 & 21.42 & 21.55 & 21.60 & 34.86 & 36.37 & 37.19 & 37.58 & 37.75 & 60.55 & 65.08 & 67.75 & 69.13 & 69.76 & 106.3 & 119.7 & 128.6 & 133.6 & 136.1 \\
\hline 2,5 & 2.25 & 12.91 & 13.00 & 13.04 & 13.06 & 13.07 & 21.16 & 21.42 & 21.55 & 21.60 & 21.62 & 36.37 & 37.19 & 37.58 & 37.75 & 37.82 & 65.08 & 67.75 & 69.13 & 69.76 & 70.03 & 119.7 & 128.6 & 133.6 & 136.1 & 137.2 \\
\hline 2,5 & 2.5 & 13.00 & 13.04 & 13.06 & 13.07 & 13.07 & 21.42 & 21.55 & 21.60 & 21.62 & 21.63 & 37.19 & 37.58 & 37.75 & 37.82 & 37.85 & 67.75 & 69.13 & 69.76 & 70.03 & 70.13 & 128.6 & 133.6 & 136.1 & 137.2 & 137.6 \\
\hline 2.5 & 2,75 & 3.04 & 13.06 & 13.07 & 13.07 & 13.07 & 21.55 & 21.60 & 21.62 & 21.63 & 21.63 & 37.58 & 37.75 & 37.82 & 37.85 & 37.86 & 69.13 & 69.76 & 70.03 & 70.13 & 70.16 & 133.6 & 136.1 & 137.2 & 137.6 & 137.8 \\
\hline 2.5 & 3 & 3.06 & 13.07 & 13.07 & 13.07 & 13.07 & \begin{tabular}{|l|l}
21.60 \\
\end{tabular} & 21.62 & 21.63 & 21.63 & 21.63 & 37.75 & 37.82 & 37.85 & 37.86 & 37.86 & 69.76 & 70.03 & 70.13 & 70.16 & 70.18 & 136.1 & 137.2 & 137.6 & 137.8 & 137.9 \\
\hline 2.5 & 3,25 & 13.07 & 13.07 & 13.07 & 13.07 & 13.07 & \begin{tabular}{|l|l|} 
\\
\end{tabular} & 21.63 & 21.63 & 21.63 & 21.63 & 37.82 & 37.85 & 37.86 & 37.86 & 37.86 & 70.03 & 70.13 & 70.16 & 70.18 & 70.18 & 137.2 & 137.6 & 137.8 & 137.9 & 137.9 \\
\hline 2.75 & 2 & .65 & 21.16 & 21.42 & 21.55 & 21.60 & 34.86 & 36.37 & 37.19 & 37.58 & 37.75 & 60.55 & 65.08 & 67.75 & 69.13 & 69.76 & \begin{tabular}{|l|l}
106.3 \\
\end{tabular} & 119.7 & 128.6 & 133.6 & 136.1 & 184.2 & 221.9 & 250.7 & 269.2 & 279.4 \\
\hline 2.75 & 2.25 & 21.16 & 21.42 & 21.55 & 21.60 & 21.62 & 36.37 & 37.19 & 37.58 & 37.75 & 37.82 & 65.08 & 67.75 & 69.13 & 69.76 & 70.03 & 119.7 & 128.6 & 133.6 & 136.1 & 137.2 & 221.9 & 250.7 & 269.2 & 279.4 & 284.2 \\
\hline 2.75 & 2.5 & & 21.55 & 21.60 & 21.62 & 21.63 & 37.19 & 37.58 & 37.75 & 37.82 & 37.85 & 67.75 & 69.13 & 69.76 & 70.03 & 70.13 & 128.6 & 133.6 & 136.1 & 137.2 & 137.6 & 250.7 & 269.2 & 279.4 & 284.2 & 286.2 \\
\hline 2.75 & 2.75 & .55 & 21.60 & 21.62 & 21.63 & 21.63 & 37.58 & 37.75 & 37.82 & 37.85 & 37.86 & 69.13 & 69.76 & 70.03 & 70.13 & 70.16 & 133.6 & 136.1 & 137.2 & 137.6 & 137.8 & 269.2 & 279.4 & 284.2 & 286.2 & 287.0 \\
\hline 2.75 & 3 & 21.60 & 21.62 & 21.63 & 21.63 & 21.63 & \begin{tabular}{|l}
37.75 \\
\end{tabular} & 37.82 & 37.85 & 37.86 & 37.86 & 69.76 & 70.03 & 70.13 & 70.16 & 70.18 & 136.1 & 137.2 & 137.6 & 137.8 & 137.9 & 279.4 & 284.2 & 286.2 & 287.0 & 287.2 \\
\hline 2.75 & 3.25 & 21.62 & 21.63 & 21.63 & 21.63 & 21.63 & 37.82 & 37.85 & 37.86 & 37.86 & 37.86 & 70.03 & 70.13 & 70.16 & 70.18 & 70.18 & 137.2 & 137.6 & 137.8 & 137.9 & 137.9 & 284.2 & 286.2 & 287.0 & 287.2 & 287.3 \\
\hline 3 & 2 & 34.86 & 36.37 & 37.19 & 37.58 & 37.75 & \begin{tabular}{|l|l}
60.55 \\
\end{tabular} & 65.08 & 67.75 & 69.13 & 69.76 & 106.3 & 119.7 & 128.6 & 133.6 & 136.1 & \begin{tabular}{|l|l}
184.2 \\
\end{tabular} & 221.9 & 250.7 & 269.2 & 279.4 & 307.1 & 404.1 & 490.9 & 556.3 & 597.6 \\
\hline 3 & 2.25 & 36.37 & 37.19 & 37.58 & 37.75 & 37.82 & \begin{tabular}{|l}
65.08 \\
\end{tabular} & 67.75 & 69.13 & 69.76 & 70.03 & 119.7 & 128.6 & 133.6 & 136.1 & 137.2 & 221.9 & 250.7 & 269.2 & 279.4 & 284.2 & 404.1 & 490.9 & 556.3 & 597.6 & 619.6 \\
\hline 3 & 2.5 & 37.19 & 37.58 & 37.75 & 37.82 & 37.85 & \begin{tabular}{|l|l}
67.75 \\
\end{tabular} & 69.13 & 69.76 & 70.03 & 70.13 & 128.6 & 133.6 & 136.1 & 137.2 & 137.6 & 250.7 & 269.2 & 279.4 & 284.2 & 286.2 & 490.9 & 556.3 & 597.6 & 619.6 & 629.6 \\
\hline 3 & 2.75 & 37.58 & 37.75 & 37.82 & 37.85 & 37.86 & \begin{tabular}{|l}
69.13 \\
\end{tabular} & 69.76 & 70.03 & 70.13 & 70.16 & 133.6 & 136.1 & 137.2 & 137.6 & 137.8 & 269.2 & 279.4 & 284.2 & 286.2 & 287.0 & 556.3 & 597.6 & 619.6 & 629.6 & 633.7 \\
\hline 3 & 3 & 37.75 & 37.82 & 37.85 & 37.86 & 37.86 & 69.76 & 70.03 & 70.13 & 70.16 & 70.18 & 136.1 & 137.2 & 137.6 & 137.8 & 137.9 & 279.4 & 284.2 & 286.2 & 287.0 & 287.2 & 597.6 & 619.6 & 629.6 & 633.7 & 635.1 \\
\hline 3 & 3.25 & 37.82 & 37.85 & 37.86 & 37.86 & 37.86 & \begin{tabular}{|l}
70.03 \\
\end{tabular} & 70.13 & 70.16 & 70.18 & 70.18 & 137.2 & 137.6 & 137.8 & 137.9 & 137.9 & 284.2 & 286.2 & 287.0 & 287.2 & 287.3 & 619.6 & 629.6 & 633.7 & 635.1 & 635.6 \\
\hline 3.25 & 2 & 60.55 & 65.08 & 67.75 & 69.13 & 69.76 & 106.3 & 119.7 & 128.6 & 133.6 & 136. & 184.2 & 221.9 & 250.7 & 269.2 & 279.4 & 307.1 & 404.1 & 490.9 & 556.3 & 597.6 & 480.9 & 702.4 & 939.2 & 1151. & 1310 . \\
\hline 3.25 & 2.25 & 65.08 & 67.75 & 69.13 & 69.76 & 70.03 & 119.7 & 128.6 & 133.6 & 136.1 & 137.2 & 221.9 & 250.7 & 269.2 & 279.4 & 284.2 & 404.1 & 490.9 & 556.3 & 597.6 & 619.6 & 702.4 & 939.2 & 1151. & 1310. & 1408. \\
\hline 3.25 & 2.5 & 67.75 & 69.13 & 69.76 & 70.03 & 70.13 & 128.6 & 133.6 & 136.1 & 137.2 & 137.6 & 250.7 & 269.2 & 279.4 & 284.2 & 286.2 & 490.9 & 556.3 & 597.6 & 619.6 & 629.6 & 939.2 & 1151. & 1310. & 1408. & 1459 \\
\hline 3.25 & 2.75 & 69.13 & 69.76 & 70.03 & 70.13 & 70.16 & 133.6 & 136.1 & 137.2 & 137.6 & 137.8 & 269.2 & 279.4 & 284.2 & 286.2 & 287.0 & 556.3 & 597.6 & 619.6 & 629.6 & 633.7 & 1151. & 1310. & 1408. & 1459 & 1481. \\
\hline 3.25 & 3 & 69.76 & 70.03 & 70.13 & 70.16 & 70.16 & \begin{tabular}{|l|}
136.1 \\
\end{tabular} & 137.2 & 137.6 & 137.8 & 137.9 & 279.4 & 284.2 & 286.2 & 287.0 & 287.2 & 597.6 & 619.6 & 629.6 & 633.7 & 635.1 & 1310 & 1408. & 1459 & 1481. & 1490. \\
\hline 3.25 & 3.25 & 70.03 & 70.13 & 70.16 & 70.16 & 70.16 & $\begin{array}{ll}137.2 \\
\end{array}$ & 137.6 & 137.8 & 137.9 & 137.9 & 284.2 & 286.2 & 287.0 & 287.2 & 287.3 & 619.6 & 629.6 & 633.7 & 635.1 & 635.6 & 1408. & 1459 & 1481. & 1490. & 1493. \\
\hline
\end{tabular}

Revista Produção Online. Florianópolis, SC, v.17, n. 3, p. 883-908, 2017. 